\title{
ANALISIS MULTIVARIADO DEL COMPLEJO DE TRES ESPECIES DE BRUNELLIA (BRUNELLIACEAE)
}

Clara Inés Orozco ${ }^{2}$

\section{RESUMEN}

Análisis de agrupamiento de componentes principales (ACP) y el árbol del valor mínimo (PRIM) fueron usados para esclarecer la sistemática de tres taxa de Brunellia: B. antioquensis (Cuatrec.) Cuatrec., B. comocladifolia Humb. \& Bonpl., y B. sibundoya Cuatrec. Fue tambien estudiada la validez de las subespecies para los dos últimos taxa. Diez y siete Unidades Taxonómicas Operacionales (UTO) representaron los taxa, y se muestrearon 32 caracteres morfológicos para cada UTO. Con base en la similitud total de los 32 caracteres se reconocen dos especies, $B$. comocladifolia y B. sibundoya, y cuatro subespecies de las seis consideradas al iniciar el trabajo. Las dos especies se encuentran a diferentes niveles altitudinales; la temperatura relacionada directamente con el gradiente altitudinal es posiblemente el factor importante en la diferenciación específica. A nivel específico los caracteres diagnósticos se refieren al número de estípulas, al indumento de los folículos y a la forma de la semilla. Los caracteres diagnósticos para el reconocimiento a nivel infraespecífico tienen que ver con el número de lobulos del cáliz y el número de carpelos; la humedad es posiblemente el factor de mayor importancia en la variación floral. Los resultados del análisis fenético muestran que $B$. antioquensis corresponde a una subespecie de $B$. sibundoya, y los taxa infraespecíficos B. comocladifolia subsp. boyacencis y B. sibundoya subsp. sebastopola son sinónimos de B. comocladifolia subsp. cundinamarcensis y $B$. sibundoya subsp. sibundoya, respectivamente. El estudio de la estructura de la exina bajo el microscopio de barrido permite tambien reconocer la variación infraespecífica. Se identifican cuatro áreas o microáreas geográficas correlacionadas con cuatro diferentes combinaciones de caracteres y que corresponden a la distribución geográfica para Colombia de los cuatro taxa infraespecíficos reconocidos bajo este estudio. El número cromosómico, $2 n=28$, es reportado por primera vez para B. comocladifolia subsp. cundinamarcensis y B. sibundoya subsp. sibundoya.

\section{ABSTRACT}

Clustering analysis, Principal Component Analysis (PCA), and Minimum Spanning Tree (PRIM) were used to define three taxa of Brunellia: B. antioquensis (Cuatrec.) Cuatrec., B. comocladifolia Humb. \& Bonpl., and B. sibundoya Cuatrec. The subspecies were studied also. Seventeen OTUs represented the three taxa, and 32 morphological characters were sampled for all OTUs. Two species, B. comocladifolia and B. sibundoya, and four subspecies instead of six were recognized. The two species are localized at different elevations. Diagnostic characters at the specific level are related to the seed shape, indumentum of follicles and stipule number. The infraspecific variation is related to the number of lobules of the calyx and the number of carpels and is associated with variation in humidity. As a result of the phenetic study, B. antioquensis is considered a subspecies of $B$. sibundoya; $B$. comocladifolia subsp. boyacensis is a synonym of subsp. cundinamarcensis; and B. sibundoya subsp. sebastopola is synonymous with $B$. sibundoya subsp. sibundoya. The pollen structure of the exine, studied using a scanning electron microscope (SEM), shows infraspecific variation also. The subspecies are found in different geographic areas, and these areas are correlated with four different character combinations. The chromosome number, $2 n=28$, is reported for the first time for $B$. comocladifolia Humb. \& Bonpl. subsp. cundinamarcensis Cuatrec. and B. sibundoya Cuatrec. subsp. sibundoya.

\footnotetext{
' Agradezco al Fondo de Investigaciones Científicas y Proyectos Especiales Francisco José de Caldas COLCIENCIAS; a Jorge Victor Crisci, Maria Fernanda López Armengol de la Universidad Nacional de La Plata, a Vicki Funk y Claudia Sobrevilla del Smithsonian Institúion por las sugerencias y críticas del manuscrito; a José Cuatrecasas con quien discutí la sistemática de los taxa y la distribución de ellas; a Amy Jean Gilmartin mis especiales agradecimientos por la prontitud en la lectura del manuscrito y sus invaluables críticas; a los profesores del Instituto de Ciencias Naturales por las sugerencias dadas al mismo. Mis agradecimientos al dibujante Silvio Fernández del Instituto de Ciencias Naturales en Bogotá; a la Bacteriologa Bertha Cova del departamento de Biología de la Universidad Nacional de Colombia por su colaboración en la preparación del material cromosómico; a Elly Beglinger de la Universidad de Amsterdam por las fotografias del grano de polen; a los curadores de los herbarios COL, MEDEL, MO, US, y VALLE; a los revisores del trabajo, José Cuatrecasas y Paul Berry por sus críticas y sugerencias. Parte de este trabajo fue presentado como requisito parcial para optar al título de Magister en Sistemática Botánica, dirigido por Enrique Forero y Jorge V. Crisci a quienes doy tambien mi agradecimiento.

${ }_{2}^{2}$ Universidad Nacional de Colombia, Instituto de Ciencias Naturales, Herbario Nacional Colombiano, Apartado Aereo 7495, Bogotá, Colombia.
}

Ann. Missouri Bot. Gard. 78: 970-994. 1991. 
Brunellia Humb. \& Bonpl. es el único género de la familia Brunelliaceae, completamente neotropical y con una amplia distribución y especiación en los bosques Andinos de Colombia. En el estado actual del conocimiento de la familia, el género comprende dos secciones y trece subsecciones (Cuatrecasas, 1970, 1985). De las 60 especies registradas actualmente para el neotrópico, 39 han sido reportadas para Colombia (Cuatrecasas, 1970, 1985; Orozco 1981, 1985, 1986).

En la revisión que se adelanta para Brunelliaceae se encontró un complejo de tres especies muy semejantes morfológicamente con superposición e inconsistencia de caracteres. Los tres taxa se encuentran ubicados en la sección Brunellia Cuatrec. subsección Comocladifoliae Cuatrec. La subsección esta definicla por la presencia de hojas opuestas e imparipinadas, foliolos con 10-17 pares de nervios secundarios, margen doble o simple, dentada, con indumento patulo. Las flores son pequeñas, y el caliz de 2.5-7.0 mm en diámetro con 4-6 piezas. $\mathrm{El}$ endocarpo es cartilaginoso en formo de $\mathrm{U}$, y el exocarpo de pelos derechos y rígidos.

El actual estudio tuvo como objetivos: establecer los límites de variación y la validez de los tres taxa conocidos como Brunellia antioquensis (Cuatrec.) Cuatrec., Brunellia comocladifolia Humb. \& Bonpl., y Brunellia sibundoya Cuatrec.; y esclarecer la existencia de subespecies cuya limitación geográfica para algunas de ellas discrepa del concepto clásico de subespecie Mayr (1963). Otro de los objetivos consistió en el estudio de caracteres no revisados anteriormente, tales como polen y cromosomas, con el propósito de conocer su utilidad y aplicación en la sistemática de la familia. Por otra parte, con este estudio se pretende aclarar los problemas encontrados por Cuatrecasas (1985) en cuanto a la determinación de las poblaciones de $B$. comocladifolic y. B. sibundoya, y provenientes de Cundinamarca, señalando entre ellas una posible hibridación.

Problemas similares a los planteados en este trabajo han sido resueltos por medio del análisis multivariado, el cual permite un conocimiento mas exacto de los caracteres.

\section{Materiales y MÉtodos}

Muestras de Brunellia antioquensis, Brunellia comocladifolic, y Brunellia sibundoya fueron coleccionadas en la región Andina colombiana, tratando de cubrir la mayor parte de ella para registrar el rango total geográfico de distribución y la variación completa de las especies. El material fue determinado con base en las claves, en la distribución geográfica, y las colecciones preexistentes en los herbarios de COL, VALLE, y MEDEL determinadas por Cuatrecasas.

En la elección de las 17 Unidades Taxonómicas Operacionales (Tabla 1) se tuvo en cuenta que las mismas representaran la variación total de los taxa y cada UTO exhibiera el estado adulto de desarrollo (en fruto). Para algunos casos las UTO están conformadas por varias muestras de un individuo y para otros casos muestras de dos o mas colecciones de varios individuos presentes en un mismo sitio. Esta última consideración fue hecha por la variación sexual exhibida en estos taxa.

Un total de 32 caracteres morfologicos florales y vegetativos fueron registrados para cada UTO (Tabla 2) de los cuales 24 son cuantitativos continuos, 3 cuantitativos discontinuos (caracteres 5, 6 , y 8), 2 cualitativos doble estado (caracteres 16 y 23) y 3 cualitativos multiestado (caracteres 30 , 31 , y 32). Varios de estos caracteres habian sido usados como diagnósticos, otros fueron elegidos teniendo en cuenta si el estado del caracter difería por lo menos en una UTO. Los caracteres de consistencia del folíolo, forma y base del folíolo, y número de nervios secundarios utilizados en previos trabajos para diferenciar los taxa, no fueron tenidos en cuenta en este estudio por la dificultad en definir con exactitud, para el caso del primer caracter, los estados coriáceo-subcoriáceo o cartáceo de moderadamente coriáceo; para el segundo y tercer caracter debido a la variabilidad intra-UTO; y para el cuarto por la ausencia de variación entre las UTO. Los caracteres cualitativos multiestados fueron codificados con base al razonamiento de una secuencia lógica de variación del caracter. Para los caracteres cuantitativos continuos el valor promedio fue anotado directamente.

La matriz básica de datos (Apéndice 1) fue sometida a distintos análisis multivariados utilizando el programa NT-SYS de Rohlf et al. (1971). Dos análisis de agrupamiento, a partir de una matriz de similitud de distancia y de una matriz de correlación; un análisis de ordenamiento (ACP); el analisis del árbol de la mínima distancia (PRIM); y los arboles de consenso fueron los análisis practicados en este trabajo (Fig. 1). En los análisis de agrupamiento, para el cálculo de la matriz de distancia, se usó el coeficiente taxonómico (TD) de Sokal (1961) y para el de la matriz de correlación el momento producto de Pearson. Las técnicas de agrupamiento del método de grupo par (Sneath \& Sokal, 1973), ligamiento promedio ponderado (WPMGA), ligamiento completo (LC), y ligamiento promedio no ponderado (UPMGA) fueron utilizadas 
TABLA 1. Relación de las UTO con sus respectivas determinaciones y referencias. Material de origen para los análisis de polen B y de cromosomas C. Cada UTO fue coleccionada en Colombia.

UTO l Brunellia comocladifolia subsp. comocladifolia. Valle del Cauca, Municipio de Dagua, Pichendé, 1,650 $\mathrm{m}$, Orozco, Lozano \& Vidal 1156 (COL) (6 especímenes).

UTO 2 Brunellia comocladifolia subsp. comocladifolia. Cauca, Municipio de Popayán, Piendamó, 1,800 m, Orozco, Vidal \& Lozano 1160 (COL), B (5 especímenes).

UTO 3 Brunellia comocladifolia subsp. comocladifolia. Valle del Cauca, Municipio, Jamundí, Villa Colombia, 1,700 m, Orozco, Vidal \& Lozano 1228 (COL) (3 especímenes).

UTO 4 Brunellia comocladifolia subsp. cundinamarcensis Cundinamarca, Municipio de Yacopi, 1,730 m, Orozco, Franco \& Lozano 1105 (COL), B, C (7 especímenes).

UTO 5 Brunellia comocladifolia subsp. cundinamarcensis. Cundinamarca, Municipio de La Palma, 1,680 m, Orozco, Franco \& Lozano 1102 (COL), B (8 especimenes).

UTO 6 Brunellia comocladifolia subsp. boyacensis. Boyacá, Municipio de La Victoria, Alto del Chapón, 1,800 $\mathrm{m}$, Orozco, Sanabria \& Sanchez 1258 (COL), C; departamento de Boyacá, Muncipio La Victoria hacia Quípama, 1,350 m, Orozco, Sanabria \& Sanchez 1267 (COL), B (1l especimenes).

UTO 7 Brunellia sibundoya subsp. sibundoya. Putumayo Valle de Sibundoy, 1,650 m, Orozco, Lozano \& Vidal 1200, 1203 (COL); 1,650 m, Bristol 1247 (COL); 2,000 m, Schultes 3202 (COL) (7 especímenes).

UTO 8 Brunellia sibundoya subsp. sibundoya. Cauca, Municipio del Puracé, Moscopán, 2,230 m, Orozco, Lozano \& Vidal 1162 (COL) (3 especimenes).

UTO 9 Brunellia sibundoya subsp. sibundoya. Cauca, Municipio del Tambo, 2,300 m, Orozco, Vidal \& Lozano 1173 (COL), B (6 especímenes).

UTO 10 Brunellia sibundoya subsp. sibundoya. Valle del Cauca, Municipio de Tulua, Barragán, 2,250 m, Orozco, Vidal \& Lozano 1120 (COL) (6 especimenes).

UTO 11 Brunellia sibundoya subsp. sebastopola. Cundinamarca, Municipio de Bojacá, 2,550 m, Orozco, Lozano \& Franco 1091, 1092, 1093 (COL), B (17 especímenes).

UTO 12 Brunellia sibundoya subsp. sebastopola. Cundinamarca, Municipio de San Francisco Sabaneta, 2,500 m, Orozco, Franco, Lozano \& Barrera 1098 (COL), C (6 especimenes).

UTO 13 Brunellia sibundoya subsp. sebastopola. Cundinamarca, Municipio de Zipacón, Estación de Sebastopol, 2,480 m, Orozco \& Carbonó 1271, 1272, 1273 (COL) (13 especímenes).

UTO 14 Brunellia antioquensis. Antioquia, Municipio de Medellín, Cerro del Boquerón, 2,420 m, Orozco, Tobón \& Henao 1320 (COL), B (6 especímenes).

UTO 15 Brunellia antioquensis. Antioquia, Municipio La Ceja, Las Lomitas, 2,170 m, Orozco, Tobon \& Henao 1290 (COL) (4 especímenes).

UTO 16 Brunellia antioquensis. Antioquia, Municipio de Medellín, Piedras Blancas, 2,250 m, Orozco, Tobón \& Henao 1280 (COL) (5 especímenes).

UTO 17 Brunellia antioquensis. Antioquia, Municipio de Rio Negro, Sajonia, 2,290 m, Orozco, Tobón \& Henao 1286 (COL), C (6 especímenes).

para agrupar los datos de similitud de cada una de las matrices (Métodos 1, 2). El coeficiente de correlación cofenética fue calculado para cada uno de los fenogramas y es el resultado de comparar la matriz de similitud, derivada del fenograma, con la matriz de similitud de la que se originaron. El uso de este coeficiente es un estimado de la forma como cada técnica de agrupamiento traduce los valores de similitud.

El análisis de los componentes principales, Método 3 , parte de una matriz de correlación entre caracteres, sin embargo, los resultados finales son las unidades taxonómicas operacionales representadas en el espacio y cuyas dimensiones corresponderían al resumen de los caracteres en vectores o componentes principales. El análisis permite tambien conocer el valor sistemático de cada uno de los caracteres. El árbol de la mínima distancia, Método 4, parte de una matriz de similitud de distancia entre UTO, y los resultados finales son las mínimas distancias fenéticas encontradas entre ellas; ver Sneath \& Sokal (1973) y Crisci \& Lopez Armengol (1983) para una mejor explicación de los procedimientos.

Los árboles de consenso (Adams, 1972) se usaron para comparar los agrupamientos obtenidos en los fenogramas; en otras palabras vienen a ser un resumen de la manera como operan las técnicas de agrupamiento para cada una de las matrices de similitud. 
TABLA 2. Caracteres utilizados en los análisis taxonómicos numéricos, registrados para cada una de las 17 UTO. Codificación de los caracteres cuantitativos discontinuos y cualitativos.

\begin{tabular}{|c|c|c|}
\hline Caracteres & Estados & $\begin{array}{l}\text { Codifi- } \\
\text { cación }\end{array}$ \\
\hline 1. Longitud de la hoja (cm) & - & - \\
\hline 2. Longitud del pecíolo $(\mathrm{cm})$ & - & - \\
\hline 3.* Longitud de los foliolos superiores $(\mathrm{cm})$ & - & - \\
\hline 4. Ancho de los foliolos superiores & - & - \\
\hline \multirow[t]{2}{*}{ 5.* Número de estípulas } & $2-3$ estipulas & 1 \\
\hline & 4-7 estipulas & 2 \\
\hline \multirow[t]{3}{*}{ 6. Número de óbulos del cáliz } & $(3) 4$ & 1 \\
\hline & $(4) 5(6)$ & 2 \\
\hline & 5 у 6 & 3 \\
\hline 7.* Diámetro celel cáliz (mm) & - & - \\
\hline \multirow[t]{3}{*}{ 8. Número de carpelos } & $(3) 4(5)$ & 1 \\
\hline & $(4) 5(6)$ & 2 \\
\hline & 5 y 6 & 3 \\
\hline 9. Separación de los nervios (mm) & - & - \\
\hline 10.* Longitud del pedicelo $(\mathrm{mm})$ & - & - \\
\hline 11. Grosor del pedicelo (mm) & - & - \\
\hline 12. Longitud de pedúnculo $(\mathrm{cm})$ & - & - \\
\hline 13. Longitud de la inflorescencia $(\mathrm{cm})$ & - & - \\
\hline 14. Longitud de foliculo $(\mathrm{mm})$ & - & - \\
\hline 15. Ancho del folículo (mm) & - & - \\
\hline \multirow[t]{2}{*}{ 16. Indumento del folículo } & Laxamente hirsuto & 1 \\
\hline & Densamente hirsuto & 2 \\
\hline 17. Ancho de la semilla (mm) & - & - \\
\hline 18. Longitud de la semilla $(\mathrm{mm})$ & - & - \\
\hline 19. Alto del endocarpo (mm) & - & - \\
\hline 20. Grosor de les ramas de primer grado de la inflorescencia (mm) & - & - \\
\hline 21. Grosor de less ramas de segundo grado de la inflorescencia $(\mathrm{mm})$ & - & - \\
\hline 22. Grosor de las ramas de tercer grado de la inflorescencia $(\mathrm{mm})$ & - & - \\
\hline \multirow[t]{2}{*}{ 23. Forma de la semilla } & Elíptica & 1 \\
\hline & Cónica & 2 \\
\hline 24. Longitud de los folíolos inferiores $(\mathrm{cm})$ & - & - \\
\hline 25. Ancho de los foliolos inferiores $(\mathrm{cm})$ & - & - \\
\hline 26. Longitud de los filamentos (mm) & - & - \\
\hline 27. Longitud de las ramas del primer grado de la inflorescencia $(\mathrm{cm})$ & - & - \\
\hline 28. Longitud de las ramas de segundo grado de la inflorescencia $(\mathrm{cm})$ & - & - \\
\hline 29. Longitud de las ramas de tercer grado de la inflorescencia (mm) & - & - \\
\hline \multirow[t]{3}{*}{ 30.* Peciolulos } & Presente & 1 \\
\hline & Presente-Ausente & 2 \\
\hline & Ausente & 3 \\
\hline \multirow[t]{3}{*}{ 31. Superficie del foliolo en estado adulto } & Liso & 1 \\
\hline & Liso o rugoso & 2 \\
\hline & Rugoso & 3 \\
\hline \multirow[t]{4}{*}{ 32.* Margen del foliolo } & $\begin{array}{l}\text { Frecuentemente serrada, } \\
\text { recta-diente delgado }\end{array}$ & l \\
\hline & $\begin{array}{l}\text { Frecuentemente serrada, } \\
\text { cóncava-diente delgado }\end{array}$ & 2 \\
\hline & $\begin{array}{l}\text { Frecuentemente serrada, } \\
\text { cóncava-diente grueso }\end{array}$ & 3 \\
\hline & $\begin{array}{l}\text { Frecuentemente crenado } \\
\quad \text { o serrado }\end{array}$ & 4 \\
\hline
\end{tabular}

\footnotetext{
* Caracteres diagnósticos en previos trabajos.
}

( ) escaso. 


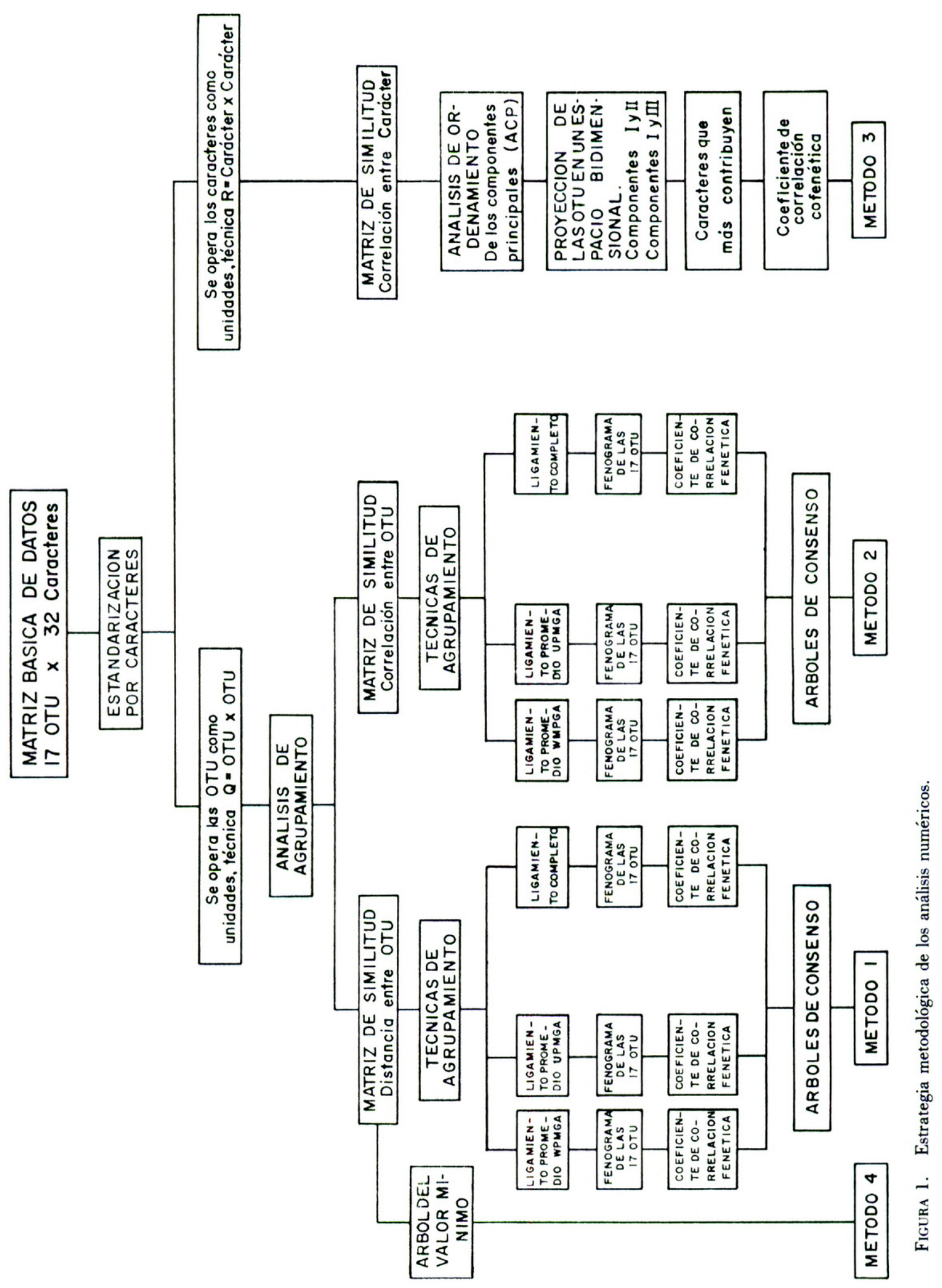


Muestras de granos de polen de las colecciones Orozco et al. 1087, 1092, 1105, 1158, 1160, $1173,1227,1257,1320,1375$, y Bernal 626 corresponden a diferentes muestras de las poblaciones (Tabla 1). Las muestras fueron enviadas al laboratorio Hugo de Vries, Amsterdam. Se utilizó la técnica Erdtman (1969) y el microscopio de barrido para obtener los micropreparados y fotografias. Directamente de las fotografias se tomaron datos del ancho del muro, ancho del lumen, longitud y ancho del grano, longitud del colpo, intercolpo y longitud y ancho del poro; estos datos fueron tambien complementados con los de las preparaciones que estaban en buen estado. Los datos del grosor de la exina se tomaron directamente de las fotografias.

Semillas de las poblaciones de las especies estudiadas, con excepción de las de Sibundoy, fueron colocadas en el germinador que consistía en una caja de petri con doble papel de filtro y agua destilada. El segundo papel de filtro fue previamente perforado en ocho huecos y en cada uno de ellos se colocaron las semillas. En estado de plántula fueron transplantadas en tierra y posteriormente llevadas al invernadero. Cuando las plantas estaban fuertes (6-7 meses de edad) se sometían a la poda de las puntas de la raíz. Este proceso se repetía cada 15 a 20 días para lograr raíces gruesas y figuras mitóticas claras en donde se pudieran contar los cromosomas. Las puntas se trataron con $\mathrm{HCl}$ $0.1 \mathrm{~N}$ y Carnoy 3:1.

\section{Resultados y Discusión}

\section{CONSIDERACIONES GENERALES}

Los resultados de los análisis de agrupamiento con los dos matrices de similitud muestran dos grandes grupos de UTO: Brunellia comocladifolia (UTO 1-6) y B. sibundoya (UTO 7-17). Cada grupo está conformado por las mismas UTO a excepción de los grupos obtenidos en el fenograma derivado de la matriz de correlación y la técnica del ligamiento completo (LC).

En todos los fenogramas se observa una mayor constancia de asociación (igual topología) para los subgrupos formados dentro del grupo de las UTO $1,2,3,4,5$, y 5 (ver consensos Figs. 3-6) mientras que para el otro grupo, integrado por las restantes UTO la constancia de asociación entre las UTO es solamente observada en los fenogramas derivados de la matriz de correlación (Figs. 5, 6). La amplia variación dentro de este último conjunto sumado al efecto propio del coeficiente de distancia son las razones por las cuales cada uno de los subgrupos resultantes en los fenogramas de distancia varian en su totalidad en el contenido de las UTO (Figs. $3,4)$. Aunque los coeficientes de correlación cofenética son ligeramente superiores a los obtenidos en los fenogramas derivados de la matriz de correlación, las verdaderas similitudes son enmascaradas. Sin embargo, se aprecia en los fenogramas de distancia la asociación constante entre las UTO 7 y $12,8,9$, y 11 y 14,16 .

El fenograma WPMGA derivado de la matriz de correlación (Fig. 2) fue escogido como el que refleja con mas exactitud las similitudes morfológicas. Este agrupamiento de las UTO da un coeficiente de correlación cofenética (C.C.C.) de 0.7017. Los dos componentes principales del análisis de los componentes principales (ACP) (Tabla 3 ) representan el $58.13 \%$ de la variación entre las UTO (Fig. 7). El árbol de la mínima distancia (PRIM) está superpuesto sobre la figura del análisis de componentes principales; las mínimas distancias estan representados por los vínculos de unión entre las UTO y por los cuales se explica el porqué de los agrupamientos obtenidos en los fenogramas de distancia.

\section{GRUPOS TAXONÓMICOS}

Los dos mayores grupos del análisis de agrupamiento corresponden a las especies Brunellia comocladifolia (UTO 1-6) y B. sibundoya (UTO 7-17); ver la Figura 2. También se observan los dos grupos por el componente I del análisis de los componentes principales (Fig. 7).

Existe una estrecha afinidad fenética entre las UTO $1,2,3$ y 4, 5, 6, formando claramente dos subgrupos que corresponden, respectivamente, a las subespecies Brunellia comocladifolia subsp. comocladifolia y B. comocladifolia subsp. cundinamarcensis. En el análisis de los componentes principales por el componente II, también se reconocen las dos subespecies (Fig. 7). La posibilidad de diferenciar a la UTO 6 como representante de una tercera subespecie boyacencis se ve bastante disminuída por cuanto existe una alta similitud fenética entre las UTO 4, 5, 6, y de ahí la constante asociación entre ellas (Figs. 3-6). Por otra parte, la similitud también fue observada en la estructura de la exina entre las subespecies cundinamarcensis y boyacensis (Fig. 9). La cercanía geográfica y la ausencia de barreras geográficas (Fig. 14) explican la variación continua de las dos poblaciones y por ende el reconocimiento de un sólo taxon $B$. comocladifolia subsp. cundinamarcensis.

Una marcada superposición fenética se observa entre las UTO que representan los taxa $B$. sibundoya subsp. sibundoya (UTO $7,8,9$ ) y $B$. si- 


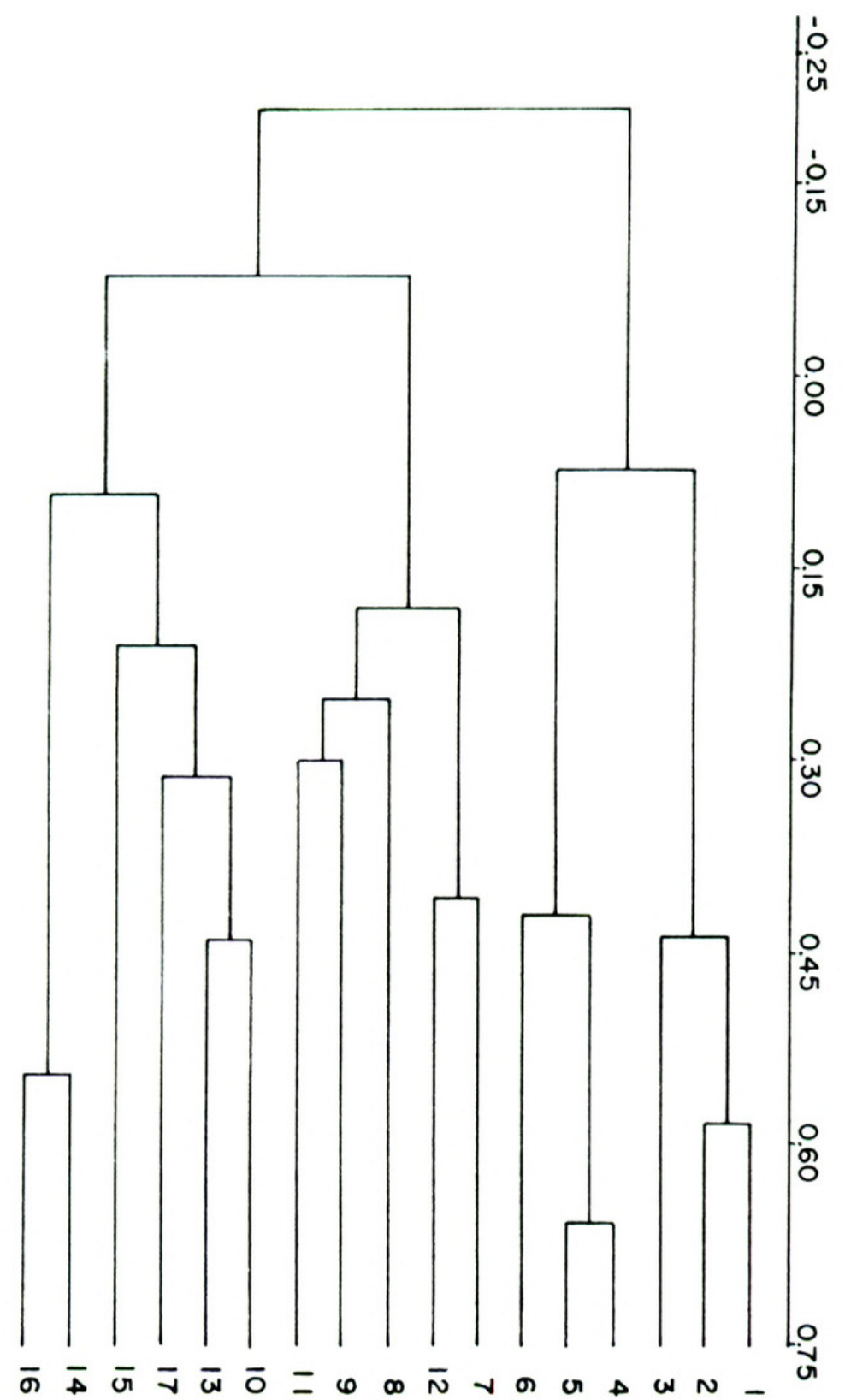

Figura 2. Fenograma de las 17 UTO basado en el coeficiente de correlación y la técnica de ligamiento ponderado WPMGA C.C.C. 0.7017 .

bundoya subsp. sebastopola (UTO 11, 12, 13). La superposición fenética es medida por la frecuente asociación en núcleos entre UTO que provienen de los diferentes grupos taxonómicos (Figs. 2-6). En el análisis de los componentes principales por el componente II, las UTO se muestran también relacionadas (Fig. 7). La estructura de la exina soporta estos resultados; no se encontró diferencia alguna en la estructura de los dos taxa (Fig. 10).

Los resultados muestran una segunda subespecie, B. sibundoya subsp. antioquensis, UTO 14 , $15,16,17$, y la UTO 10 (Figs. 2, 5-7). La UTO 13, en los análisis de correlación está relacionada con B. sibundoya subsp. antioquensis pero por el análisis de los componentes principales se encuen- tra mas asociada con B. sibundoya subsp. sibundoya.

En la Figura 7 (cuadrantes derecho, inferior y superior), las interconexiones debidas a las mínimas distancias fenéticas entre las UTO de los dos taxa infraespecíficos B. sibundoya son el resultado de una mayor relación fenética entre las subespecies que la encontrada en $B$. comocladifolia; en la misma figura cuadrante izquierdo inferior y superior se observa sólo una interconexión, entre las UTO 1 y 6 , por tratarse de una unidad específica. Bajo diferente concepto taxonómico que el mostrado por los resultados de este trabajo, Cuatrecasas (1985) anota una estrecha afinidad entre B. sibundoya y $B$. antioquensis. 
Tabla 3. Eigen-valores. Porcentaje de traza. Acumulación de porcentaje. Análisis de los componentes principales (ACP).

\begin{tabular}{lccc}
\hline \hline Componentes principales & $\begin{array}{c}\text { Valores propios } \\
\text { Eigen-Valores }\end{array}$ & \% de Traza & Acumulación de \% \\
\hline Primer componente & 14.185 & 44.33 & 44.33 \\
Segundo componente & 4.417 & 13.80 & 58.13 \\
Tercer componente & 3.527 & 10.44 & 68.57 \\
Cuarto componente & 2.267 & 6.71 & 75.28 \\
Quinto componente & 1.963 & 5.81 & 81.09 \\
Sexto componente & 1.518 & 4.49 & 85.58 \\
Séptimo componente & 1.334 & 3.95 & 89.53 \\
Octavo componente & 0.987 & 92.45 \\
Noveno componente & 0.696 & 2.92 & 94.51 \\
Décimo componente & 0.485 & 1.43 & 95.94 \\
Décimo primer componente & 0.398 & 0.84 & 96.78 \\
Décimo segdo. componente & 0.308 & 0.91 & 97.53 \\
Décimo tercer componente & 0.255 & 0.75 & 98.28 \\
Décimo cuarto componente & 0.180 & 0.53 & 98.81 \\
Décimo quinto componente & 0.153 & 0.45 & 99.26 \\
Décimo sexto componente & 0.118 & 0.34 & 99.60 \\
Décimo síptimo componente & 0.037 & 0.10 & 99.70 \\
Décimo octavo componente & 0.026 & 0.07 & 99.77 \\
Décimo noveno componente & 0.016 & 0.047 & 100.24 \\
\hline
\end{tabular}

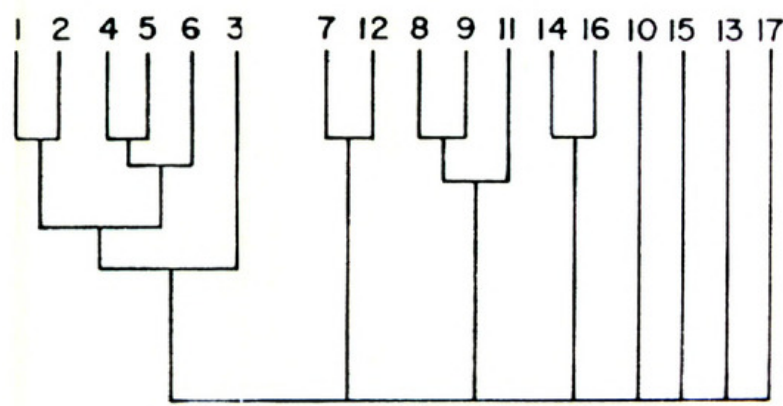

(3)

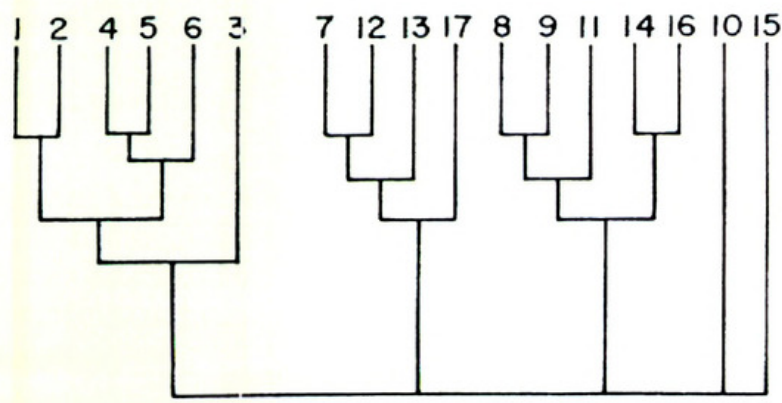

(4)

Figuras 3 y 4 . Consenso entre las técnicas de agrupamiento basadas en el coeficiente de distancia. - 3. Consenso Estricto. - 4. Consenso de Mayoria.

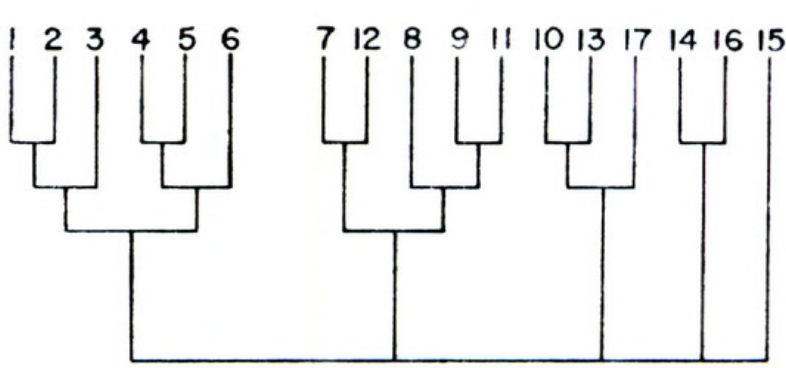

(5)
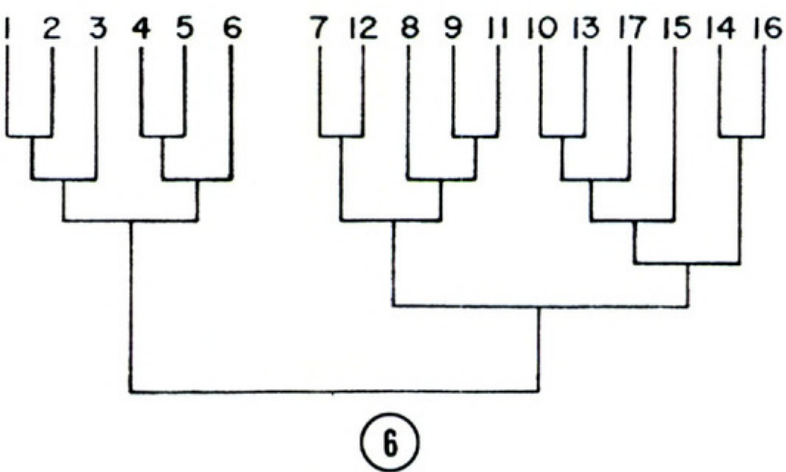

Figuras 5 y 6 . Consenso entre las técnicas de agrupamiento basadas en el coeficiente de correlación. -5 . Consenso Estricto. -6. Consenso de Mayoria. 


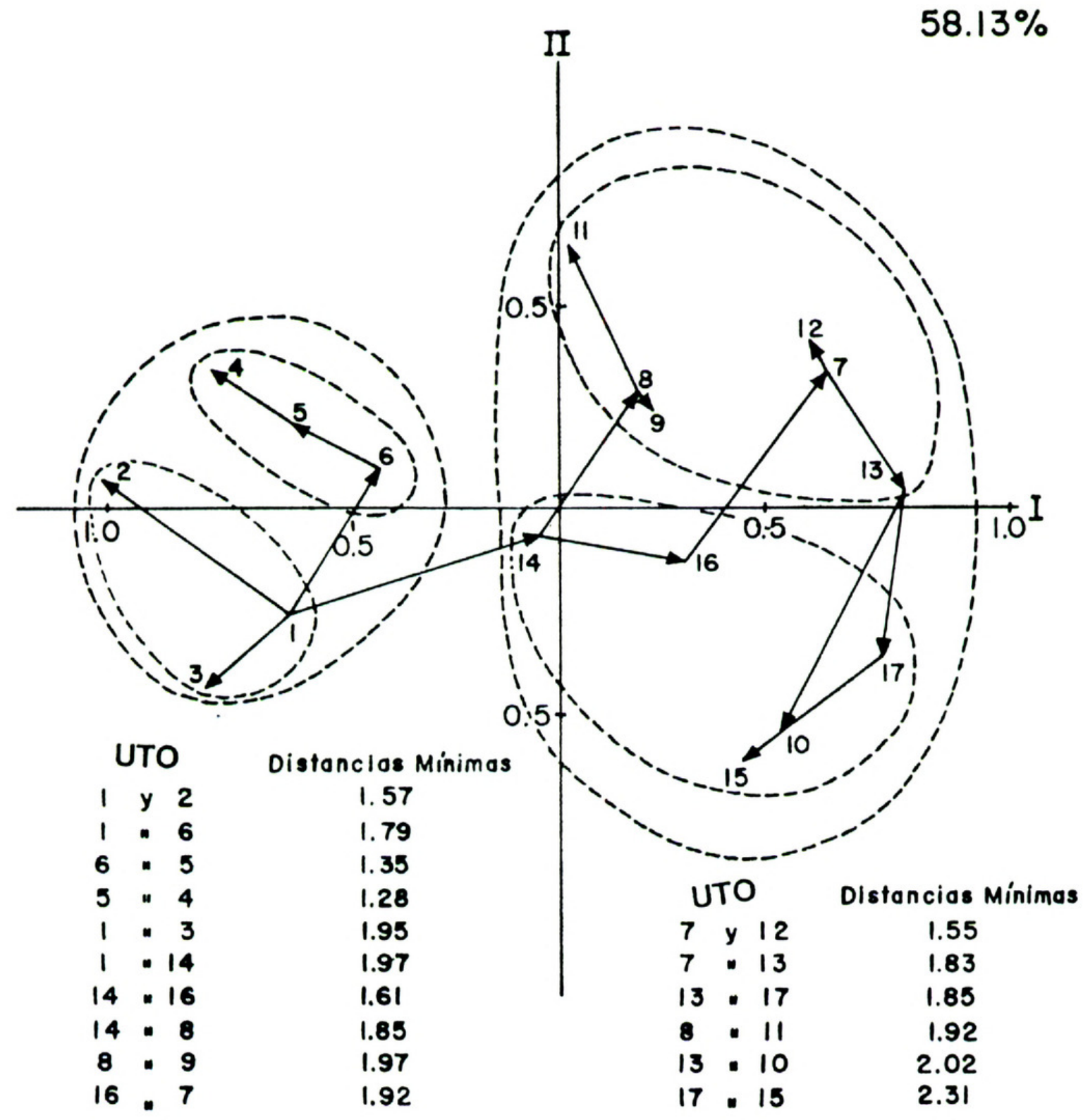

Figura 7. Análisis de los componentes principales (ACP) y el árbol del valor mínimo (PRIM). Las líneas entre las UTO representan las distancias mínimas entre ellas.

Los agrupamientos de distancia entre las UTO que integran las dos subespecies de Brunellia sibundoya (Figs. 3,4) así como las interconexiones fenéticas son debidas a una amplia variación de los caracteres cuantitativos continuos. Asi, los extremos superiores e inferiores de estos caracteres son compartidos por las UTO de los dos diferentes grupos taxonómicos infraespecíficos, por ejemplo las mayores longitudes promedio de los folíolos superiores $(15.16-19.04 \mathrm{~cm})$ son exhibidas por las UTO 10, 15, 17 (B. sibundoya subsp. antioquensis) y las UTO 13, 12 (B. sibundoya subsp. sibundoya); las menores longitudes (9.69-12.30 $\mathrm{cm})$ se encuentran en las UTO 14,18 (B. sibundoya subsp. antioquensis) y las UTO 8, 7, 9, 11 (B. sibundoya subsp. sibundoya). En la Figura 7 estas UTO se encuentran unidas por las distancias fenéticas mínimas.

CARACTERES DISCRIMINANTES PARA EL

RECONOCIMIENTO DE LOS

GRUPOS TAXONÓMICOS

En el análisis de los componentes principales (Fig. 7), el componente I separa a los taxa Brunellia comocladifolia y Brunellia sibundoya. De los 32 caracteres morfológicos 3 de ellos (caracteres $23,10,5$ ) en el componente I son importantes para diferenciar las especies (Tabla 4). La forma de la semilla en $B$. comocladifolia es elíptica, el indumento sobre los folículos es laxo y el número de estípulas no es superior a 3 (Figs. 16, 17). En 
contraposición, la semilla en $B$. sibundoya es cónica, el indumento sobre los folículos es denso, y el número de estípulas es mayor de 3 (Figs. 18, 19).

Los caracteres cuantitativos continuos del grosor de las ramificaciones de la inflorescencia (caracteres $20,21,22)$ y el ancho de los folíolos superiores e inferiores (caracteres 4,25 ), cuyos aportes al componente I son superiores a 0.8 , no son muy útiles como diąnósticos por cuanto hay superposición de los límites superiores e inferiores. Sin embargo, en promedio el grosor de las ramificaciones y el ancho de los foliolos son menores en B. comocladifolia y pueden considerarse como tendencias. El ancho de los folíolos está relacionado con la forma lanceolada o angostamente oblonga observada con relativa frecuencia en $B$. comocladifolia. De manera similar en B. sibundoya los caracteres de grosor de las ramificaciones y ancho de los foliolos pueden considerarse como tendencia hacia mas gruesos y anchos, respectivamente. El ancho de los foliolos tiene que ver con las formas ovada, largamente ovada, ampliamente elíptica o ampliamente oblonga de los folíolos; estados observados con frecuencia en cada unidad taxonómica (UTO).

Dos caracteres $(6,8)$ en el componente II son útiles para la diferenciación infraespecífica (Tabla 4): B. comocladifolia subsp. comocladifolia exhibe 4 lóbulos del cáliz y 4 carpelos, en contraposición con la subespecie cundinamarcensis con 5 lóbulos del cáliz y 5 carpelos. En B. sibundoya subsp. sibundoya se encuentra con mayor frecuencia 5 lóbulos del cáliz y 5 carpelos, pero también se observó en algunas muestras hasta 6 lóbulos del cáliz y 6 carpelos. Brunellia sibundoya subsp. antioquensis se reconoce por la frecuencia de 4 lóbulos del cáliz y 4 carpelos y con menos frecuencia 5 lóbulos del cáliz y 5 carpelos.

Tres aspectos de los caracteres diagnósticos son importantes de mencionar en el momento: (1) los caracteres se encuentran correlacionados con las áreas o microáreas geográficas ocupadas por los taxa infraespecificos (Fig. 15); (2) existe una alta correlación (análisis de agrupamiento de caracteres, técnica R) entre los caracteres diagnósticos tanto para la diferenciación específica como infraespecífica (Orozco, 1989); y (3) el escaso número de caracteres diagnósticos está relacionado con la estrecha afinidad morfológica entre las dos especies.

\section{ESTRUCTURA DE LA EXINA}

No se encontraron diferencias significantes en el tamaño del छrano de polen, longitud del colpo,
TABLA 4. Análisis de los componentes principales. Aporte de cada uno de los caracteres a los componentes principales.

\begin{tabular}{|c|c|c|c|}
\hline \multirow{2}{*}{$\begin{array}{c}\text { Carac- } \\
\text { teres }\end{array}$} & \multicolumn{3}{|c|}{ Componentes } \\
\hline & l & 2 & 3 \\
\hline l & 0.541 & 0.491 & 0.461 \\
\hline 2 & 0.735 & 0.349 & 0.493 \\
\hline 3 & 0.595 & 0.373 & 0.596 \\
\hline 4 & 0.855 & 0.270 & 0.271 \\
\hline 5 & 0.908 & 0.089 & 0.208 \\
\hline 6 & 0.107 & 0.880 & 0.323 \\
\hline 7 & 0.658 & 0.170 & 0.430 \\
\hline 8 & 0.187 & 0.791 & 0.344 \\
\hline 9 & 0.644 & 0.634 & 0.154 \\
\hline 10 & 0.699 & 0.065 & 0.020 \\
\hline 11 & 0.626 & 0.348 & 0.399 \\
\hline 12 & 0.630 & 0.430 & 0.377 \\
\hline 13 & 0.033 & 0.399 & 0.066 \\
\hline 14 & 0.731 & 0.230 & 0.358 \\
\hline 15 & 0.663 & 0.072 & 0.251 \\
\hline 16 & 0.906 & 0.049 & 0.243 \\
\hline 17 & 0.532 & 0.343 & 0.318 \\
\hline 18 & 0.255 & 0.207 & 0.277 \\
\hline 19 & 0.681 & 0.025 & 0.072 \\
\hline 20 & 0.852 & 0.139 & 0.182 \\
\hline 21 & 0.868 & 0.246 & 0.131 \\
\hline 22 & 0.930 & 0.139 & 0.066 \\
\hline 23 & 1.008 & 0.028 & 0.170 \\
\hline 24 & 0.754 & 0.130 & 0.519 \\
\hline 25 & 0.884 & 0.049 & 0.311 \\
\hline 26 & 0.507 & 0.427 & 0.114 \\
\hline 27 & 0.397 & 0.418 & 0.023 \\
\hline 28 & 0.377 & 0.264 & 0.452 \\
\hline 29 & 0.730 & 0.316 & 0.168 \\
\hline 30 & 0.467 & 0.720 & 0.210 \\
\hline 31 & 0.268 & 0.251 & 0.827 \\
\hline 32 & 0.741 & 0.186 & 0.072 \\
\hline
\end{tabular}

intercolpo, y longitud y ancho del poro. Las diferencias se observan a nivel de la estructura de la exina.

Brunellia comocladifolia exhibe una estructura reticulada con tendencia en la subespecie comocladifolia a lúmenes más amplios y muros continuos y aplanados (Fig. 8). La subespecie cundinamarcensis exhibe una exina reticulada irregular con lúmenes menos amplios y muros parcialmente fracturados y realzados (Fig. 9).

Se observa en B. sibundoya dos patrones diferentes de la estructura de la exina. Uno de ellos corresponde a la subespecie sibundoya con una estructura perforada rugulosa (Fig. 10); el otro patron a la subespecie antioquensis con una estructura reticulada irregular (Fig. 11) y muy similar a la observada en B. comocladifolia subsp. cundinamarcensis. 


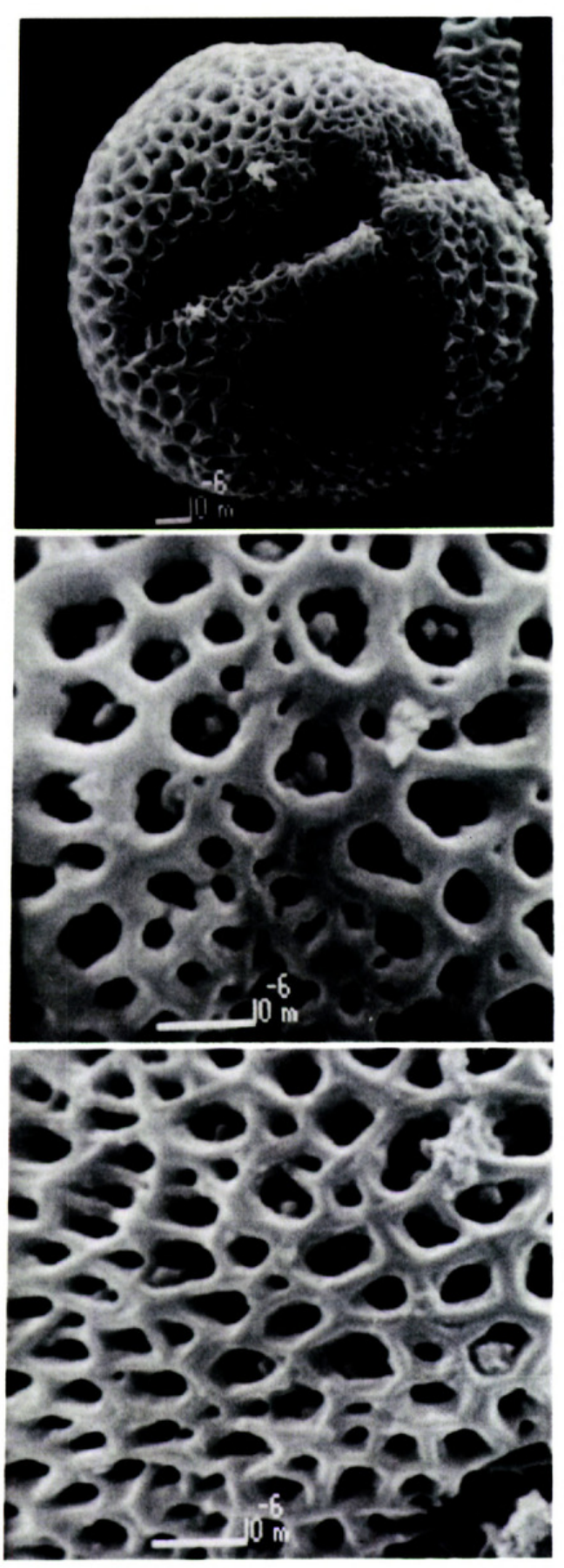

a

Figura 8. Forma y estructural del grano del polen de Brunellia comocladifolia Humb. \& Bonpl. subsp. comocladifolia. a y b, Orozco et al. 1227; c, Orozco et al. 1158.

Cuatrecasas (1985) se refiere también a las variaciones infraespecíficas de la estructura de la exina encontradas por Marticorena (1970), bajo el microscopio de luz, en otras subespecies neotropicales de B. comocladifolia. Muller (1979) y Nowicke \& Skvarla (1979), quienes han observado las variaciones estructurales de la exina a nivel infraespecífico, atribuyen dicha variación a un amplio componente de adaptación del grano de polen, especialmente el de la superficie del tectum. Po- siblemente también sea el caso de la variación encontrada en Brunellia.

\section{CROMOSOMAS}

El número cromosómico $2 n=28$ es reportado para Brunellia comocladifolia subsp. cundinamarcensis y B. sibundoya subsp. sibundoya. Para las dos otras subespecies no se reporta el número cromosómico debido a que las plántulas no tuvieron éxito. Los resultados presentados fueron obtenidos sin pretratamiento alguno (Figs. 12, 13). Las diferencias en el tamaño de los cromosomas se deben a diferentes estadios metafásicos.

Los datos del número de cromosomas coinciden con los reportados por Ehrendorfer et al. (1984) para Brunellia mexicana Standl. y B. comocladifolia subsp. funckiana (Tul.). Ehrendorfer et al. (1984) señalan la dificultad en obtener un cariotipo claro, dificultad también observada en este estudio por la escasa precisión en la morfología de los cromosomas.

\section{DISTRIBUCIÓN GEOGRÁFICA}

Las dos subespecies de Brunellia comocladifolia se encuentran en las selvas subandinas entre $1,000-1,800 \mathrm{~m}$, mientras que $B$. sibundoya se registra a alturas mayores entre $2,000-2,900 \mathrm{~m}$, ocupando las selvas andinas y subandinas.

Brunellia comocladifolia subsp. comocladifolia no sólo se distribuye en el sur de Colombia y Ecuador (Cuatrecasas, 1985) sino que se extiende hacia el norte por la cordillera occidental hasta Antioquia. Brunellia comocladifolia subsp. cundinamarcensis se distribuye sobre la cordillera oriental y se reporta para el norte en el Huila, Cundinamarca y en Boyacá; posiblemente se extiende más hacia el sur y más hacia el norte en los departamentos de Santander. Los datos disponibles para el estudio permiten visualizar la separación geográfica entre las dos subespecies por la cordillera central (Fig. 14). Se infiere que la diferenciación infraespecífica pudo haber ocurrido en el sur de Colombia en los sitios geográficos que corresponden a la ramificación de los Andes colombianos.

Brunellia sibundoya subsp. sibundoya tiene una más amplia distribución (Fig. 14). Original. mente la subespecie fue reportada en el Nudo de los Pastos (Putumayo), en la cordillera central flanco oriental, e interconexiones con la cordillera occidental, Cauca (Cuatrecasas, 1970), sitios donde se registraron las menores alturas $(2,000-2,300$ $\mathrm{m})$. Nuevas colecciones en el Tolima señalan el ascenso de la subespecie a lo largo de la vertiente 

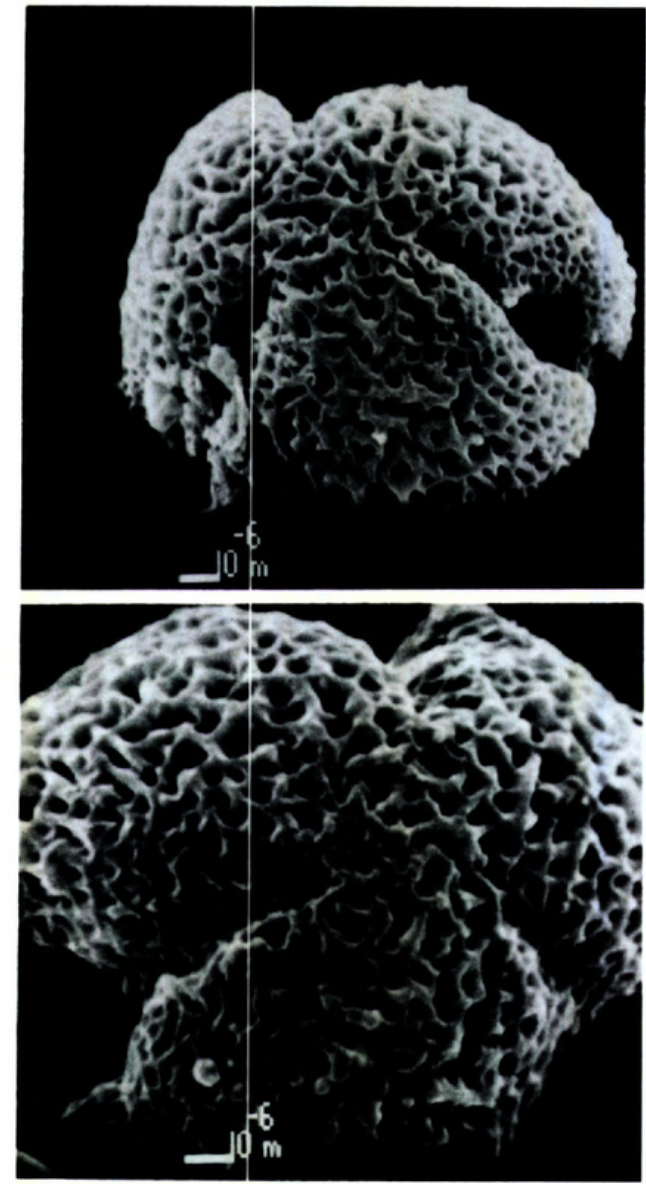

a

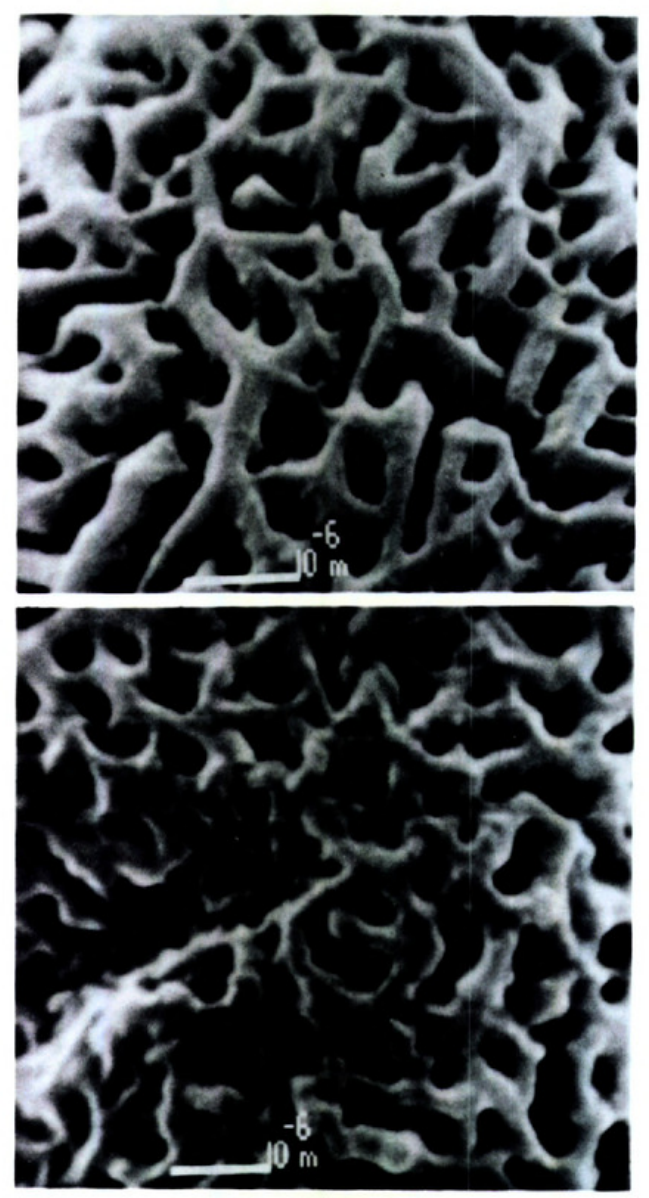

Figura 9. Forma y estructura del grano del polen de Brunellia comocladifolia Humb. \& Bonpl. subsp. cundinamarcensis. Cuatrec. a y b, Orozco et al. 1105; c y d, Orozco et al. 1267, colección muestreada como Brunellia comocladifolia subsp. boyacensis.

oriental de la cordillera central. Los resultados del actual estudio indican también la presencia del taxon en la cordillera oriental (Cundinamarca) y por los datos disponibles se infiere que la presencia de la subespecie en la cordillera oriental se deba más bien al ascenso por la misma desde el Nudo de los Pastos. Esta inferencia se apoya en el registro del taxon para el sur del Huila en los límites con el departamentos del Cauca, cercano al lugar del nacimiento del ramal de la cordillera oriental y la separación entre la cordillera central y oriental (Río Magdalena, Fig. 14).

En contraposición con los restantes subespecies los datos disporibles permiten reconocer el taxon B. sibundoya subsp. antioquensis en una área geográfica mas estrecha y con una amplia distribución hacia el norte de las cordilleras central y occidental. El taxon en previos trabajos fue registrado hacia el norte en la cordillera central (Antioquia), sitios en donde la cordillera no tiene sus más altas elevaciones; nuevas colecciones indican su distribución en la cordillera occidental (Caldas y Antioquia) y más hacia el sur en la cordillera central-vertiente occidental (Valle del Cauca y Caldas).
La presencia del taxon en la cordillera occidental y en la cordillera central puede estar relacionada con la diferenciación infraespecífica en el sur de Colombia o, por la subespeciación mas hacia el norte, en la cordillera central. Esta última pudo haber sido favorecida por la disimetría climática en los dos flancos de la cordillera a causa de los efectos de la glaciación (Salomons, 1986). Bajo esta consideración, debe de haber existido una amplia dispersión por su ocurrencia en la cordillera occidental. Las subespecies antioquensis y sibundoya se encuentran en los sitios más elevados de la cordillera central y donde las vertientes son más acentuadas (Fig. 14).

$\mathrm{Al}$ parecer la diferenciación específica está relacionada con el gradiente altitudinal, mientras que la infraespecífica lo está con la cantidad de humedad. Estos dos factores climáticos (Sarmiento, 1986) vienen a ser los factores de mayor importancia ecológica variando a través y a lo largo de las cordilleras andinas. La humedad, que caracteriza a la cordillera occidental y vertiente occidental de la cordillera central, aunque debida a diferentes causas tiene que ver con la reducción 

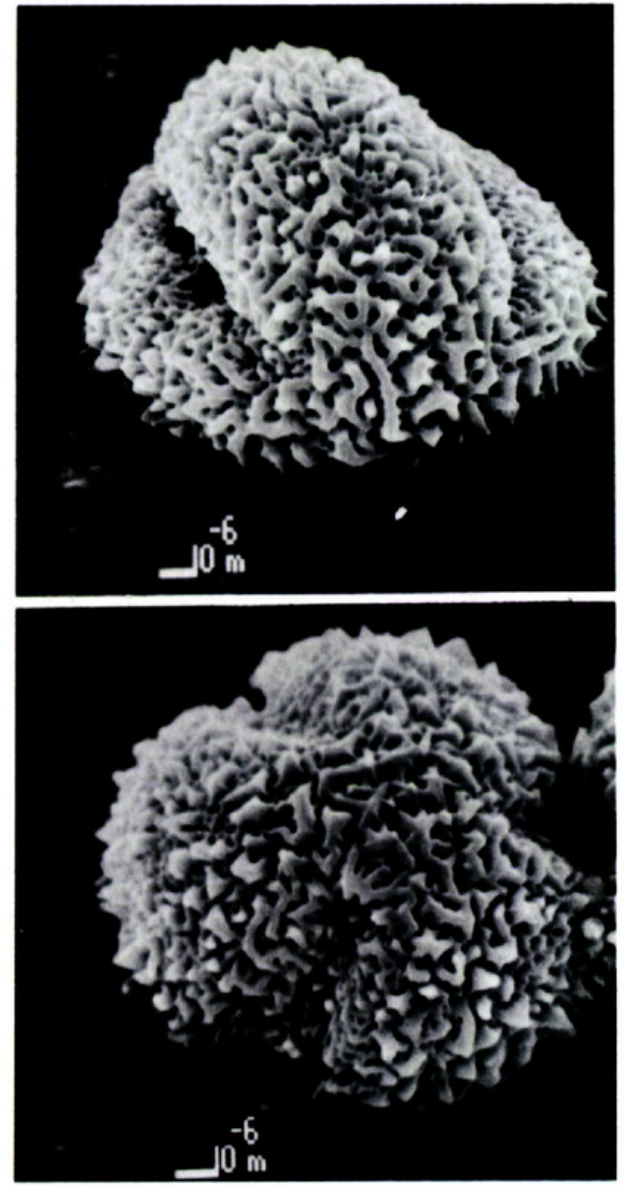

a

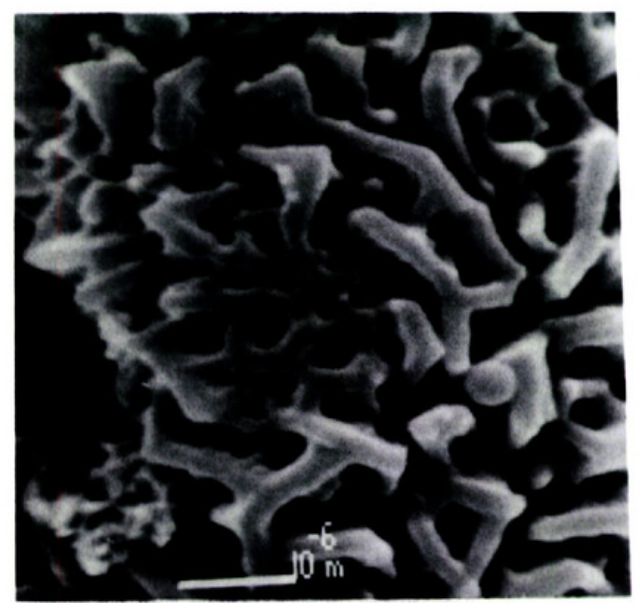

$\mathrm{b}$

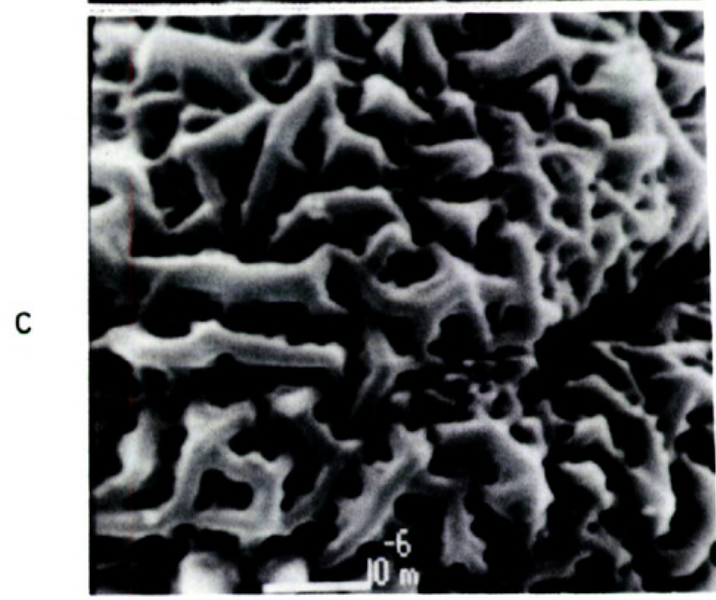

Figura 10. Forma y estructura del grano de polen de Brunellia sibundoya Cuatrec. subsp. sibundoya. a y b, Orozco et al. 1173; c y d, Orozco et al. 1087, colección muestreada como Brunellia sibundoya subsp. sebastopola.

del número de partes florales: B. comocladifolia subsp. comocladifolia y B. sibundoya subsp. antioquensis tienen solamente cuatro lóbulos del cáliz y cuatro carpelos.

Los datos de distribución permiten establecer cuatro áreas o microáreas geográficas libres de superposición y correlacionadas con cuatro diferentes combinaciones de caracteres morfológicos y de estructura de la exina (Fig. 15).

\section{Tratamiento Sistemático}

Clave para las Especies y Subespecies de BRUNELLIA ESTUDIADAS

1a. Estípulas 2-3; indumento del folículo laxamente hirsuto; semilla elíptica; 1,000-1,800 m.

2a. Lóbulos del cáliz y carpelos 4 ; cordillera occidental e interconexiones al sur de las cordilleras central y occidental.

la. B. comocladifolia subsp. comocladifolia

2b. Lóbulos del cáliz y carpelos 5; cordillera oriental …….... lb. B. comocladifolia subsp. cundinamarcensis

1b. Estípulas 4-7; indumento del folículo densamente hirsuto; semilla cónica; 2,000-2,800 m.

3a. Lóbulos del cáliz y carpelos 5-6; en el sur del Macizo colombiano e interconexiones de las cordilleras occidental y central y vertiente oriental de la cordillera central 2a. B. sibundoya subsp. sibundoya

3b. Lóbulos del cáliz y carpelos 4(5); hacia el norte en la cordillera occidental y vertiente occidental de la central 2b. B. sibundoya subsp. antioquensis

1. Brunellia comocladifolia Humb. \& Bonpl. Pl. Aequinoct. 1: 211-213, pl. 59. 1808; HBK Nov. Gen. Sp. 7: 46. 1825; Cuatrec. Fl. Neotropica, 2: 63-68. 1970; Supplement 43-46. 1985.

Arbol 5-12 m. Estípulas 2-3. Hojas compuestas opuestas, 12.4-73.2 cm de largo, imparipinadas 1-7 yugos, 3.15(-18) foliolos; pecíolo 3.3-14.0 cm de largo. Folíolos peciolulados o sésiles, los superiores 3.6-16.0 cm de largo, (1.4-)2.0-7.4 cm de ancho, oblongo-elípticos, elípticos, lanceolados; base del foliolo obtuso o cuneado. Folíolos inferiores ovados (1.7-)2.5-10.5 cm de largo, (1.0-)1.6-5.0 cm de ancho; márgen de los folíolos crenado-serrada, serrada o márgen cóncavo-serrada; nervios secundarios prominentes por el envés; $\left(6^{-}\right) 9-18(-20)$ pares, distantes entre si $(1.5-) 3.0^{-}$ 
$18 \mathrm{~mm}$; indumento sobre los nervios de pelos largos, flexibles. Superficie de los foliolos lisa y menos frecuente rugosa. Inflorescencia tirsoide, 6.3-30.0 cm de largo; ramificaciones delgadas, en cimas dicasiales, isotónicas, ramas de primer grado 1.2$30 \mathrm{~mm}$ de grueso, de segundo grado 1.0-2.0($3.0) \mathrm{mm}$ de grueso, de tercer grado de $0.8-1.5(2.0)$ mm de grueso. Pedúnculo $1.5-12.5 \mathrm{~cm}$ de largo. Flores hermafroditas o unisexuales, pediceladas en fruto, en flores femeninas y con menos frecuencia en flores masculinas; pedicelos 2.0-3.5 $\mathrm{mm}$ de largo, 0.2-0.5(--1.0) mm de grueso. Cáliz pentámero o tetrámero, diámetro del cáliz en fruto (3.5-)4.0-5.5(-6.0) mm. Estambres 10, filamentos (1.5-)2.0-3.0 rnm de largo. Carpelos 4 o 5. Folículos 4 o 5 , con indumento laxamente hirsuto, ovados, 2.2-3.0 $\mathrm{mm}$ de largo, 2.0-3.0 $\mathrm{mm}$ de ancho. Endocarpo seco en forma de U, 2.0-2.7(2.9) $\mathrm{mm}$ de alto. Semilla 1 desarrollada por folículo, elíptica, 1.8-2.2 $\mathrm{mm}$ de largo, $1.5-2.0 \mathrm{~mm}$ de ancho.

1a. Brunellia comocladifolia Humb. \& Bonpl. subsp. comocladifolia Fl. Neotropica 2: 6667. 1970; Supplement 43-45. 1985. TIPO: Colombia. Cauca: Popayan, 1906, Humboldt \& Bonplarid s.n. (holótipo, $\mathrm{P}$; isótipo, $\mathrm{P}$ n.v.). Figura 16.

Arbol 5-12 m. Folíolos peciolulados 1.0-4.0 $\mathrm{mm}$ de largo; foliolos inferiores de una misma rama de menor tamaño comparados con los superiores; margen de los folíolos en su máximo desarrollo tendiente a ser cóncava y serrada. Cáliz tetrámero. Carpelos 4. Folículos 4 con muy escasa frecuencia en menor número. Granos de polen con exina regularmente reticulada.

Distribución Brunellia comocladifolia subsp. comocladifolia tiene una amplia distribución desde el sur (Ecuador), y a lo largo de de la cordillera occidental en Colombia, hasta el departamento de Antioquia. También al sur en la intersección entre la cordillera central y la occidental.

Ejemplares representativos. Colombia. ANTIOQUiA: Municipio La Concordia, vía Urrao, 1,750 m, 3 dic. 1984 (fr), Orozco, Tobón \& Henao 1341 (COL). RISARalda: Municipio de Pueblo rico, carretera al cerro Montezuma, 1,350 m, 5 mar 1986 (fl o), Bernal, Galeano, Tobón \& Henao 1101 (COL). VALLE DEL CAUCA: cordillera occidental, hoya del río Calima, El Cairo entre Darién y Mediacanoa, 1,650-1,750 m, 7 ene. 1943 (fr), Cuatrecasas 13867 (COL); Municipio de Dagua, Pichindé, 1,650 m, 22 sep. 1983 (fr), Orozco, Vidal \& Lozano 1156 (COL).
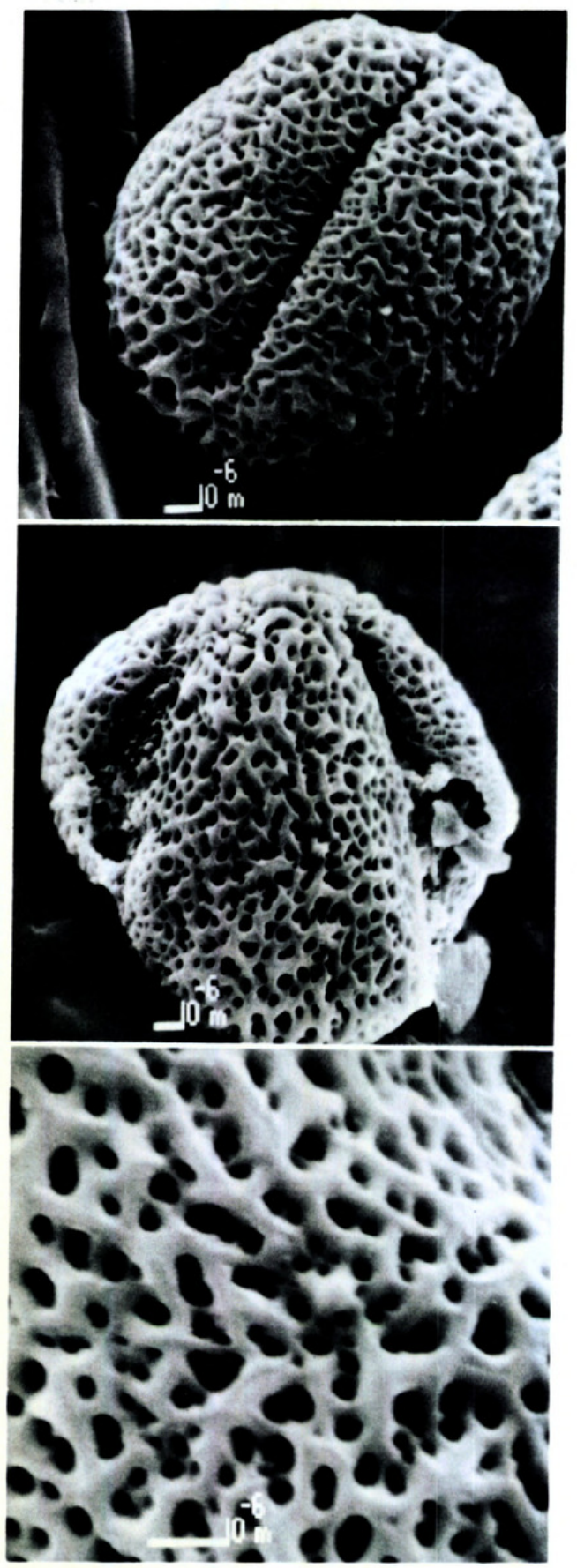

Figura 11. Forma y estructura del grano de polen de Brunellia sibundoya subsp. antioquensis Cuatrec. a, Orozco et al. 1320 ; b y c, Orozco et al. 259 , colecciones muestreadas como Brunellia antioquensis.

1b. Brunellia comocladifolia Humb. \& Bonpl. subsp. cundinamarcensis Cuatrec. Fl. Neotropica 2: 68-69. 1970; Supplement 45. 1985. TIPO. Colombia. Cundinamarca: Susumuco, 1,000 m, dic. 1855 (f), Triana 3720 (holótipo, W; isótipos, BM, G, US, W). Figura 17.

Brunellia comocladifolia Humb. \& Bonpl. subsp. boyacensis Cuatrec. Fl. Neotropica 2: 67-68. 1970. TIPO: Colombia. Boyacá: Monte del Chapón, 1,230 


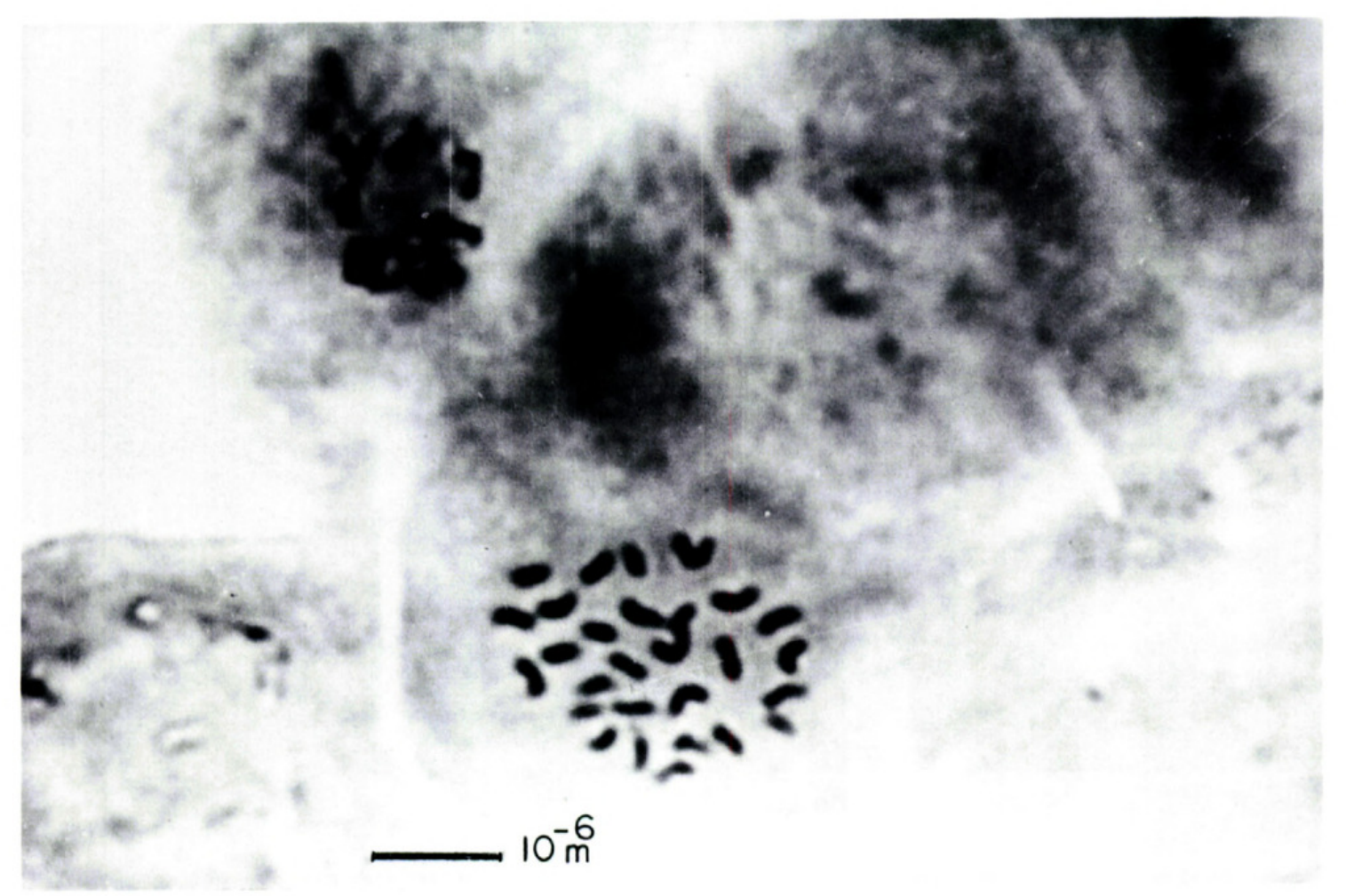

Figura 12. Cromosomas metafásicos de Brunellia sibundoya subsp. sibundoya.

m, 21 jul. 1932 (fr), Lawrence 347 (holótipo, NY; isótipos, BM, F, G, GH, MO, S, U, US).

Arbol 6-10 m. Foliolos sésiles o muy cortamente peciolulados, 0-1.5 mm de largo, base obtusa o cuneada, margen serrada o crenado-serrada. Lóbulos del cáliz 5. Carpelos 5. Granos de polen con exina irregularmente reticulada.

Distribución. Cordillera oriental en los departamentos de Cundinamarca, Boyacá y en Huila.
Existe en COL un especimen de la colección Little 7253 procedente de Huila que parece más bien corresponder por los datos de altura, 2,000-2,030 $\mathrm{m}$, a B. sibundoya subsp. sibundoya.

Ejemplares representativos. Colombia. Boyaca: Municipio La Victoria, Alto del Chapón, 29 jul. 1984 (fr), Orozco, Sanabria \& Sanchez 1256 (COL); hacia la quebrada de la Colichona, vereda San Martín, 1,500 m, 29 jul. 1984 (fr), Orozco, Sanabria \& Sanchez 1265 (COL); hacia Quípama, 1,350 m, 30 jul. 1984 (fl, o), Orozco,

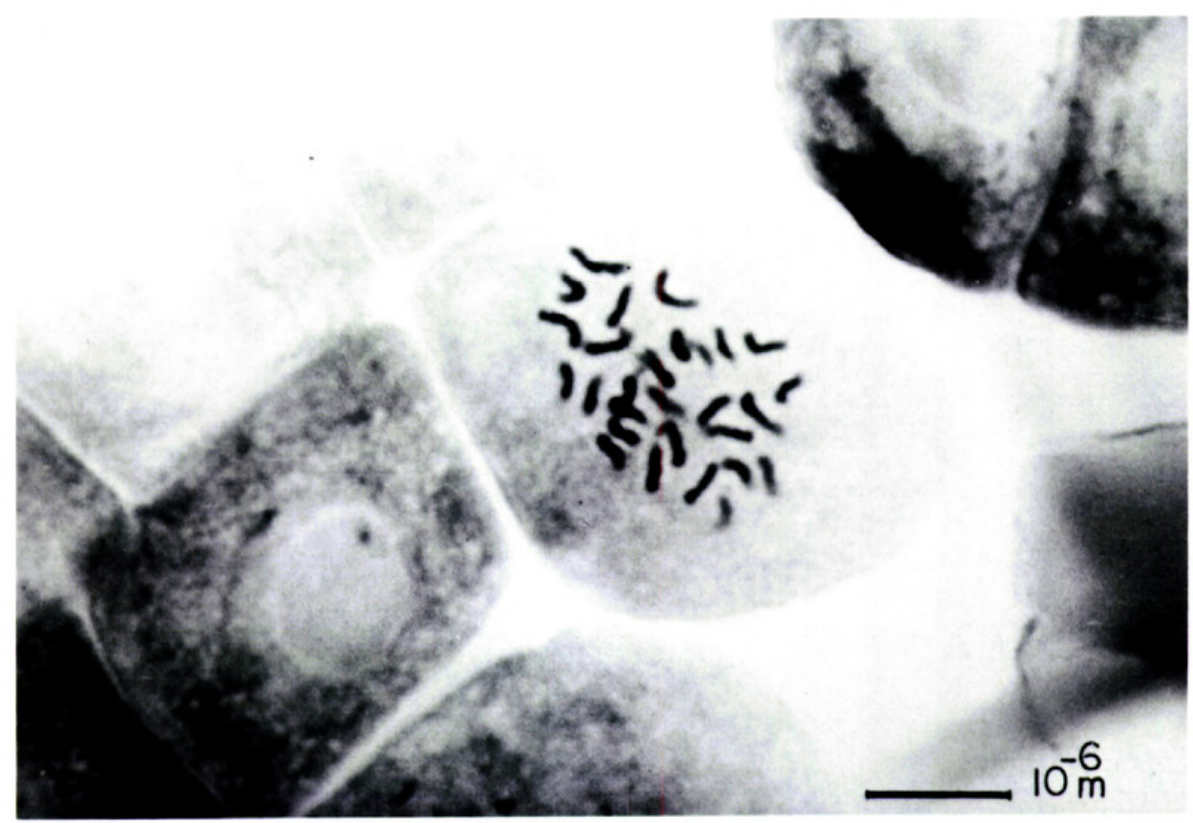

Figura 13. Cromosomas premetafásicos de Brunellia comocladifolia subsp. cundinamarcensis. 


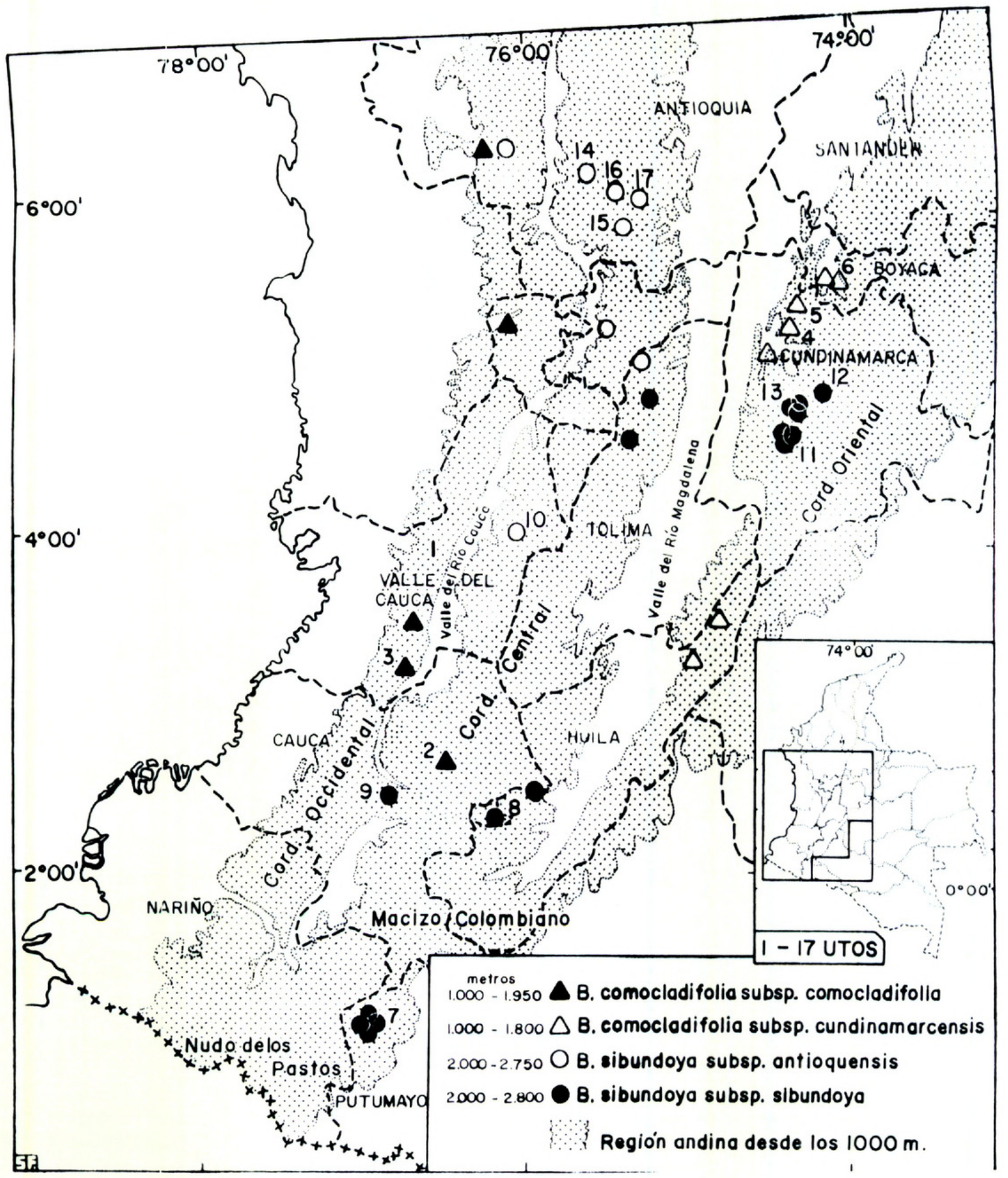

Figura 14. Distribución geográfica de Brunellia comocladifolia Humb. \& Bonpl. Brunellia sibundoya Cuatrec. Los números se refieren a las UTO.

Sanabria \& Sanchez 1267 (COL). Cundinamarca: Municipio de la Palma, 1,730 m, 30 mayo 1983, Orozco, Lozano \& Franco 1102 (COL); Sasaima, 1,600 m, 35 ene. 1973 (fl, o), García Barriga 20379 (COL). HUILA: Río Venadito, $25 \mathrm{~km}$ SE de la Bodega, 1,780 m, 2 dic. 1944 (fr), Little 9024 (COL).

Nombre vulgar. Tambor, en Cundinamarca y Boyacá.
2. Brunellia sibundoya Cuatrec. Revista Acad. Colomb. Ci. Exact. 5: 35. 1942; Cuatrec. Fl. Neotropica 2: 78-79. 1970; Supplement 4648. 1985.

Arbol 6.0-20 m. Estípulas 4-7. Hojas compuestas opuestas, (9.0-)20-74.3(-83.8) $\mathrm{cm} \mathrm{de}$ 


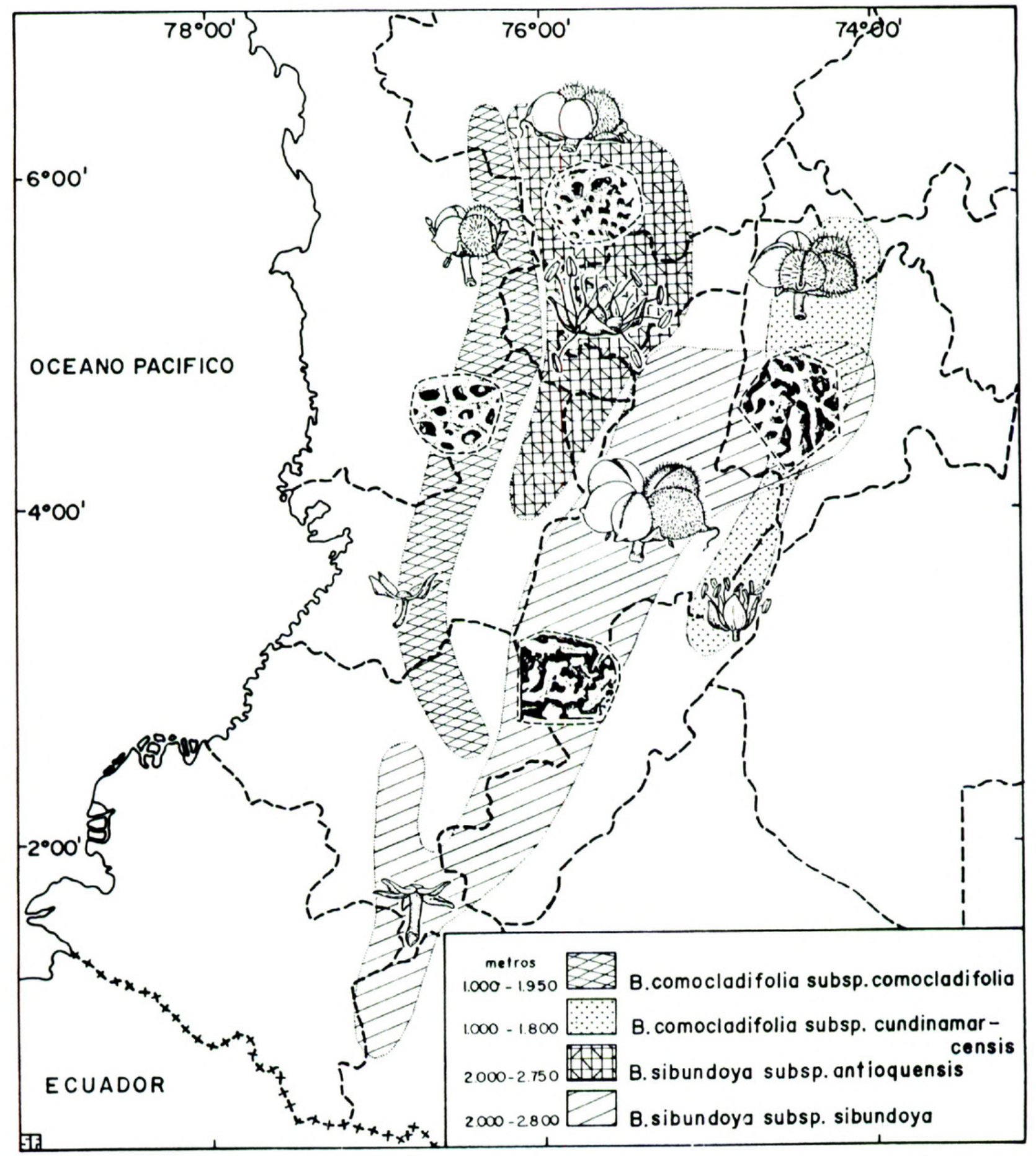

Figura 15. Distribución geográfica y caracteres diagnósticos de las especies de Brunellia estudiadas.

largo, imparipinadas, 1-7 yugos, 3-15 foliolos. Pecíolo 3.0-19.5 cm de largo. Folíolos sésiles o peciolulados, los superiores $6.0-28.0 \mathrm{~cm}$ de largo, (2.5-)3.3-10.6 cm de ancho, ovados, ovadooblongos, elípticos u oblongo-elípticos; base obtusa o cuneada; margen de los foliolos crenado-serrada o cóncavo-serrada; nervios secundarios prominentes por el envés, (6-)9-22 pares, se apartan entre sí (2.0)3.0-21.0 mm. Folíolos inferiores ovados; $4.3-17.0 \mathrm{~cm}$ de largo, $1.8-9.9 \mathrm{~cm}$ de ancho. Superficie de los foliolos lisa o rugosa. Inflorescen- cia en tirso o en dicasio, 6.5-31.0 cm de largo; ramificaciones gruesas, en dicasios de una casi perfecta isotonía, ramas de primer grado, $(1.5-)\left(2.0_{-}^{-}\right.$ 4.2) (-5.5) $\mathrm{mm}$ de grueso, de segundo grado (1.0-)2.0-3.5 mm de grueso, de tercer grado $1.2-$ $3.0(-4.0) \mathrm{mm}$ de grueso. Pedúnculo $1.5-18.0 \mathrm{~cm}$ long. Flores hermafroditas o unisexuales, pediceladas en fruto o en flores femeninas, sésiles en flores masculinas, pedicelos $1.0-2.0(-2.5) \mathrm{mm}$ de largo, $(0.8-)(1.0-1.5)(-2.0) \mathrm{mm}$ de grueso. Cáliz pentámero o tetrámero (hexámero), diámetro en 

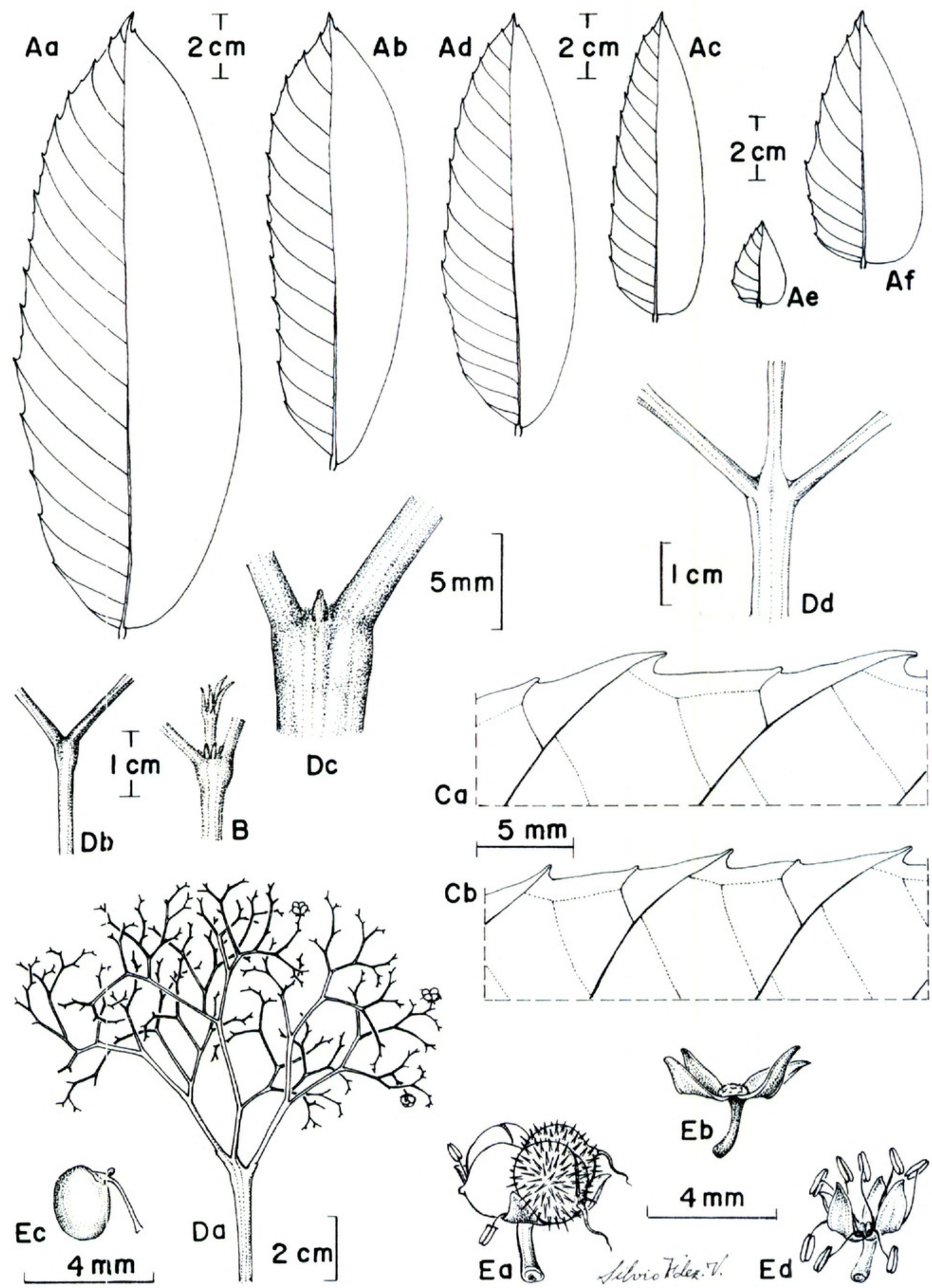

Figura 16. Brunellia comocladifolia Humb. \& Bonpl. subsp. comocladifolia. A. Variación en forma y tamaño de los folíolos. - Aa-Ad. Folíolos superiores. - Ae, Af. Folíolos inferiores. - B. Detalle de las estípulas y yema. - C. Margen del foliolo. - Da. Inflorescencia completa. - Db-Dd. Ramificación de la inflorescencia. - E. Partes de la flor y fruto. - Ea. Folículos y estambres. - Eb. Número de lóbulos del cáliz. - Ec. Semilla. - Ed. Flor masculina. A (a, d, f), B, C (b), D (a-d), y E (a-c) Orozco et al. 1156; A (b), Orozco et al. 1160; A (c), Duque-Jaramillo 3893; A (e) y C (a), Cuatrecasas 13857; E (d), Orozco et al. 1158.

fruto (5.0-)5.5-7.0(-7.5) mm. Estambres 10, filamentos (2.0-i2.8-5.0 $\mathrm{mm}$ de largo. Folículos 4 o 5(6), con indumento denso e hirsuto, ovados, $2.8-4.0(-4.2) \mathrm{mm}$ de long, $(2.2-) 3.0-4.0 \mathrm{~mm}$ de ancho. Endocarpo seco en forma de U, (2.0-)2.5$3.0(-4.0) \mathrm{mm}$ de alto. Semilla 1 desarrollada por foliculo, cónica, (1.2-)2.0-2.5(-3.0) $\mathrm{mm}$ de largo, (1.2-)1.8-2.0(-2.2) $\mathrm{mm}$ de ancho. 


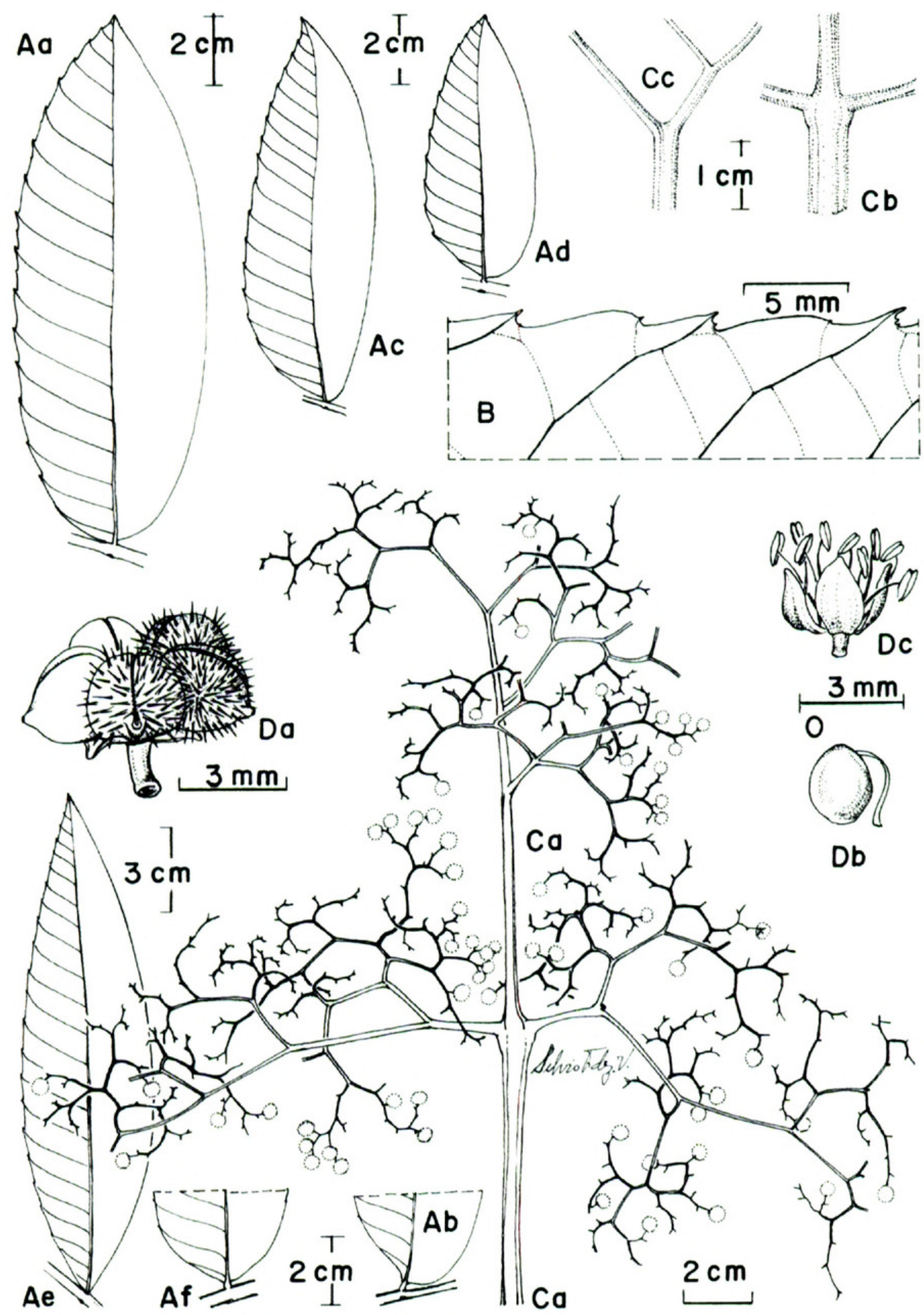

Figura 17. Brunellia comocladifolia Humb. \& Bonpl. subsp. cundinamarcensis Cuatrec. - A. Forma, tamaño de los foliolos y estado del pecíolulo. - B. Margen del folíolo.-Ca. Inflorescencia completa. - Cb, Cc. Ramificación de la inflorescencia. D. Partes de la flor y fruto. - Da. Foliculos. - Db. Forma de la semilla. - Dc. Flor masculina. A (a, b), C (a-c), y D (a, b), Orozco et al. 1102; A (c, d), Garcia-Barriga 20379; A (e, f), y D (c), Orozco et al. $1267 ;$ B, Orozco et al. 1251.

2a. Brunellia sibundoya Cuatrec. subsp. sibundoya Fl. Neotropica 2: 80. 1970; Supplement 46. 1985. TIPO: Colombia. Comisaría del Putumayo: Cuenca alta del río Putumayo en el Valle de Sibundoy, $2,200 \mathrm{~m}, \mathrm{l}$ ene. 1941 (fr), Cuatrecasas 11570 (holótipo, US; isótipos, F, US). Figura 18.
Brunellia sibundoya Cuatrec. subsp. sebastopola. Fl. Neotropica 2: 81. 1970; supplement 46-48. 1985. TIPO: Colombia. Cundinamarca: cordillera oriental, entre Sebastopol y Alto de las Escaleras, 2,3002,400 m, 21 mar. 1942 (fl, ठ), Cuatrecasas 13586 (holótipo, US; isótipos, COL, F).

Arbol 8-20 m. Folíolos sésiles, generalmente 

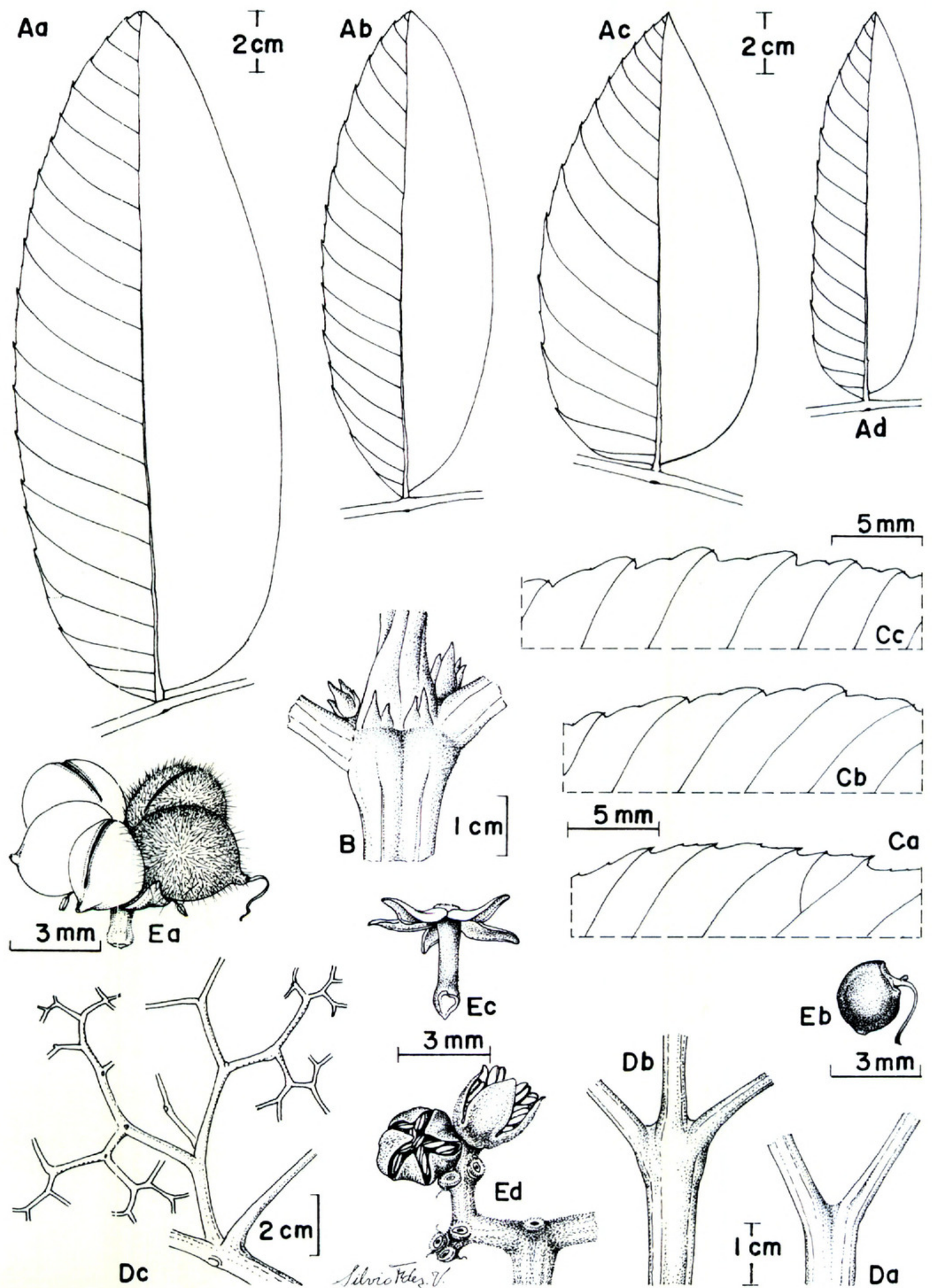

Figura 18. Brunellia sibundoya Cuatrec. subsp. sibundoya. - A. Forma y tamaño de los foliolos. - B. Detalle de las estípulas.--C. Margen del foliolo.-Da, Db. Ramificación de la inflorescencia.-Dc. Inflorescencia completa. E. Partes de la for y fruto. - Ea. Folículos. - Eb. Forma de la semilla. - Ec. Número de lóbulos del cáliz. - Ed. Flor masculina. A (a), D (a, b), y E (a-c), Orozco et al. 1273; A (b), Cuatrecasas 11570; A (c), Schultes 3202; A (d), Orozco et al. 239; C (a), Vidal 113; C (b), Idrobo et al. 10325; C (c) y E (d), Cuatrecasas 13586; D (c), Bristol 1273. 

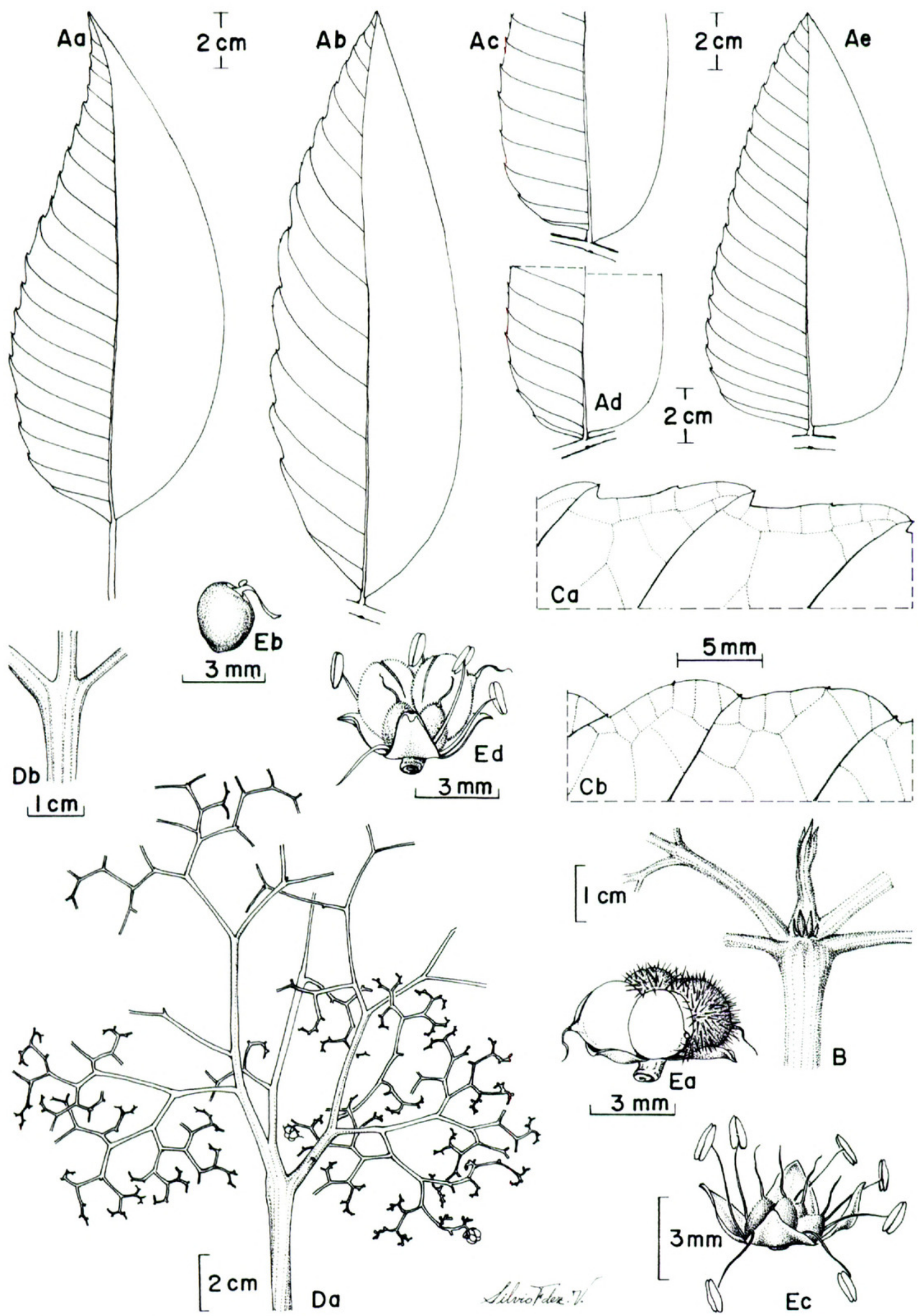

Figura 19. Brunellia sibundoya Cuatrec. subsp. antioquensis Cuatrec.-A. Forma, tamaño de los folíolos y estado del peciólulo (a, foliolo terminal). - B. Detalle de las estípulas. - C. Detalle de la margen del foliolo. - Da. Inflorescencia completa. - Db. Ramificación de la inflorescencia. - E. Partes de la flor y fruto. - Ea. Folículos. - Eb. 
crenado-serrados, con menos frecuencia dentados. Cáliz 5-6 lóbulos. Carpelos 5-6. Folículos todos desarrollados. Granos de polen con exina perforada rugulosa.

Distribución. Se registra desde el Macizo colombiana hasta Cundinamarca en la cordillera oriental y para el Tolima en la cordillera central.

Ejemplares representativos. Colombia. CUNDINAMarCa: Municipio de San Francisco, Vereda las Minas, 2,600 m, may. 1981 (fr), Orozco \& Barrera 239 (COL); Municipio de Zipacón, Sebastopol, 2,480 m, 20 nov. 1984 (fr), Orozco \& Carbonó 1273 (COL); cerros de los alrededores de Bogotá, 2,700 m, 11 jun. 1962 (fl, ơ), Saravia 1207 (COL). TOLimA: El silencio, Juntas, 2,400 m, 8 dic. 1984 (ir), Vidal s.n. (COL); Municipio Santa Isabel, Vereda Lc. Yuca, Alto La Esperanza, 2,700 m, 11 jul. 1980 (fl), Idrobo, Cleef, Rangel \& Salamanca 10325 (COL). Putumayo: Valle de Sibundoy, $2,200 \mathrm{~m}$, 19 jul. 1963 (fr), Bristol 1247 (COL); 2,250 m, 12 feb. 1942 (fr), Schultes 3202 (COL).

El isótipo estudiado de Brunellia sibundoya subsp. sebastopola es un especimen de flores masculinas y foliolos delgados. La condición sésil observada en previos trabajos y utilizada como caracter diagnóstico para diferenciar las subspecies sebastopola y sibundoya es constante en las inflorescencias masculinas. La condición delgada de los foliolos utilizada también como caracter diagnóstico representa solo un estado de la variación.

2b. Brunellia sibundoya subsp. antioquensis Cuatrec. Fl. Neotropica 2: 85. 1970. Brunellia antioquensis (Cuatrec.) Cuatrec. Fl. Neotropica 2; Supplement 48. 1985. TIPO: Colombia. Antioquia: Cerca de Santa Elena, entre Medellín y Río Negro, 2,300-2,500 m, (fl, fr), Gutierrez \& Deslisle 1126 (holótipo, $\mathrm{F}$; isótipo, F). Figura 19.

Arbol 6-15 m. Folíolos sésiles o cortamente peciolulados, crenado-serrados o fuertemente dentados. Cáliz 4 lóbulos, menos frecuente 5. Carpelos 4 , raro 5. Folículos todos desarrollados. Granos de polen con exina irregularmente reticulada.

Distribución. Se extiende hacia el norte de Colombia en las cordilleras occidental y central (vertiente occidental). Se registra en los departamentos del Valle del Cauca, Caldas, y Antioquia.

Ejemplares representativos. COLOMBIA. ANTIOQUIA: Municipio de Giraldo, carretera a Urabá, Boquerón de
Toyo, 2,100 m, 19 mayo 1983 (fr), Bernal 626 (COL); Municipio de Medellín, cerro del Boquerón, 2,420 m, 29 nov. 1984 (fr), Orozco, Tobón \& Henao 1320 (COL); Municipio de Río Negro, Sajonia, 2,290 m, 27 nov. 1984 (fr), Orozco, Tobón \& Henao 1286 (COL); Municipio de Santa Fé de Antioquia, Cañasgordas, Boquerón de Toyo, 2,000 m, 18 feb. 1985 (fl, ơ), Henderson \& Bernal 145 (COL); Municipio de Urrao, Alto de Caicedo, 2,270 m, 4 dic. 1984 (fl, ơ, fr), Orozco, Tobón \& Henao 1355 (COL). Caldas: San Antonio de Padua, 2,430 m, 6 oct. 1981 (f), Orozco, Rivera, Torres, Pinto \& Lozano 259 (COL).

\section{Conclusiones}

De los tres taxa considerados al iniciar el estudio sólo dos especies, Brunellia comocladifolia y Brunellia sibundoya, se reconocen por los análisis de agrupamiento, componentes principales (ACP) y el árbol de la mínima distancia (PRIM). Los dos taxa son el resultado de los estudios de 17 Unidades Taxonómicas Operacionales y 32 caracteres morfológicos.

Los caracteres diagnósticos para reconocer las dos especies son en escaso número. Sin embargo, los taxa son fenéticamente distintos con base en la similitud total de los 32 caracteres. Los caracteres cuantitativos continuos como el ancho de los foliolos, directamente relacionado con la forma (Orozco, 1989), y el grosor de las ramificaciones de las inflorescencias pueden ser considerados como tendencias en el reconocimiento de las especies, mas no como caracteres diagnósticos por presentar superposición de los límites de variación.

Las diferentes interconexiones fenéticas (PRIM) observadas entre las unidades taxonómicas que integran B. sibundoya, y los agrupamientos de distancia entre diferentes miembros de las dos subespecies son atribuídas a la variación de los caracteres cuantitativos continuos (24 en total). Posiblemente, la amplia distribución del taxon está relacionada con la plasticidad fenótipica de estos caracteres. Para B. comocladifolia se registra una mayor homogeneidad medida por la constante asociación entre las UTO que integran cada una de las subespecies; en otras palabras siempre se encuentran las mismas UTO conformando los grupos infraespecíficos, por otra parte en el PRIM se encuentra sólo una interconexión entre miembros de los dos grupos infraespecíficos.

Mientras que la temperatura en relación con el gradiente altitudinal es un factor determinante para

Forma de la semilla.-Ec. Flor con estambres y carpelos poco desarrollados. - Ed. Folículos y estambres. A (a), C (b), D (a), y E (a, b), Orozco et al. 1286; A (b) y E (c), Henderson et al. 145; A (d), Orozco et al. 1289; B, Orozco et al. 1186; C (a), Bernal et al. 626; D (b), Orozco et al. 1290; E (d), Orozco et al. 1320. 
la diferenciación específica, la humedad es posiblemente para el caso de la variación infraespecífica el factor predominante. Los taxa infraespecíficos con la tendencia hacia el menor número de partes florales están distribuídos en las zonas de mayor humedad, la cordillera occidental y la vertiente occidental de la cordillera central.

Por los datos de distribución se infiere que la diferenciación infraespecífica está muy estrechamente relacionada con la bifurcación de los ramales de la cordillera de los Andes en el Sur de Colombia. Sin embargo, la subespeciación para el caso de $B$. sibundoya se registró hacia el norte de la cordillera central.

\section{Literatura Citada}

Adams, E. N. 1972. Consensus techniques and the comparison of taxonomic trees. Syst. Zool. 21: 390397.

Crisci, J. V. \& M. F. Lopez Armengol. 1983. Introducción a la Teoría y Práctica de la taxonomía numérica. Serie de biología: Monogr. 26. Organización de los Estados Americanos, Washington, D.C.

Cuatrecasas, J. 1970. Brunelliaceae. Fl. Neotrop. Monogr. 2.

- 1985. Brunelliaceae. Fl. Neotrop. Monogr. 2. [Supplement.]

Ehrendorfer, F., W. Morawetz \& J. Dawe. 1984. The neotropical angiosperm families Brunelliaceae and Caryocaraceae: first karyosystematical data affinities. Pl. Syst. Evol. 145: 183-191.

Erdtman, G. 1969. Handbook of Palynology. Hafner, New York.

Marticorena, C. 1970. Pollen morphology. Pp. 2732 in J. Cuatrecasas, Brunelliaceae. Fl. Neotrop. Monogr. 2.
MAYr, E. 1963. Especies Animales y Evolución. Ediciones Univ. Chile, Ariel, Chile.

Muller, J. 1979. Form and function in angiosperm pollen. Ann. Missouri Bot. Gard. 66: 593-632.

NowiCKe, J. \& J. Skvarla. 1979. Pollen morphology: the potential influence in higher order systematics. Ann. Missouri Bot. Gard. 66: 633-700.

Orozco, C. I. 1981. Contribución al estudio de las Bruneliaceas de Colombia-I. Mutisia 50: 4-8.

- 1985. Dos especies nuevas de Brunellia de Colombia. Pp. 20-24 in J. Cuatrecasas, Fl. Neotrop. Monogr. 2. [Supplement.]

-. 1986. Contribución al estudio de Brunelliaceae de Colombia. III. Caldasia 15: 176-185.

- 1989. Correlación de caracteres para algunas especies de Brunellia. Revista Acad. Colomb. Ci. Exact. 17(65): 357-365.

Pearson, K. 1926. On the coefficient of racial likeness. Biometrika 18: 105-117.

Rohlf, F., J. KishPaugh \& D. KIRK. 1971. NT-SYS. Numerical taxonomy system of multivariate statistical programs. Techn. Rep. State Univ. New York, Stony Brook, New York.

Salomons, J. B. 1986. Pp. 157-171 in Paleoecology of Volcanic Soils in the Colombian Central Cordillera (Parque Nacional Natural de los Nevados). J. Cramer, Berlin \& Stuttgart.

SARmiEnto, G. 1986. Ecological features of climate in high tropical mountains in high altitude tropical biogeography. Pp. 11-45 in Vuillemier \& Monasterio (editors), High Altitude Tropical Biogeography. Oxford Univ. Press, New York.

Sneath, P. \& R. Sokal. 1973. Numerical Taxonomy: The Principles and Practice of Numerical Classification. Freeman, San Francisco.

SOKAL, R. 1961. Distance as a measure of taxonomic similarity. Syst. Zool. 10: 70-79. 
APÉnDICE I. Matriz básica de datos. Información obtenida para cada Unidad Taxonómica Operacional (UTO). UTO

\begin{tabular}{|c|c|c|c|c|c|c|c|c|c|c|c|c|c|c|c|c|c|}
\hline & & 2 & 3 & 4 & 5 & 6 & 7 & 8 & 9 & 10 & 11 & 12 & 13 & 14 & 15 & 16 & 17 \\
\hline 1 & 34.95 & 22.92 & 31.52 & 24.50 & 35.62 & 45.17 & 30.84 & 26.83 & 29.70 & 46.30 & 34.15 & 31.11 & 61.50 & 34.00 & 57.30 & 31.93 & 51.22 \\
\hline 2 & 8. $2 ! 5$ & 4.70 & 7.06 & 6.17 & 7.69 & 9.84 & 7.16 & 9.06 & 9.59 & 14.97 & 9.09 & 11.11 & 12.30 & 7.29 & 11.36 & 8.61 & 13.00 \\
\hline 3 & 12.34 & 12.68 & 9.98 & 9.09 & 12.01 & 13.60 & 12.30 & 12.30 & 11.67 & 19.04 & 11.51 & 15.16 & 17.78 & 11.53 & 16.20 & 9.90 & 15.24 \\
\hline 4 & 4.80 & 2.41 & 3.84 & 3.16 & 4.20 & 4.23 & 6.44 & 4.77 & 4.61 & 6.90 & 4.44 & 5.23 & 6.71 & 3.79 & 6.17 & 4.61 & 6.22 \\
\hline 5 & 1 & 1 & 1 & 1 & 1 & 1 & 2 & 2 & 2 & 2 & 2 & 2 & 2 & 2 & 2 & 2 & 2 \\
\hline 6 & 1 & 1 & 1 & 2 & 2 & 2 & 2 & 2 & 2 & 1 & 3 & 2 & 2 & 1 & 1 & 1 & 1 \\
\hline 7 & 5.84 & 4.83 & 5.22 & 4. 27 & 4.03 & 3.93 & 6.14 & 5.42 & 6.45 & 6.12 & 5.40 & 5.75 & 5.87 & 6.59 & 5.20 & 5.93 & 6.61 \\
\hline 8 & 1 & 1 & 1 & 2 & 2 & 2 & 2 & N.C & 2 & 1 & 2 & 3 & 2 & 1 & 1 & 1 & 1 \\
\hline 9 & 8.513 & 6.36 & 8.18 & 6.26 & 7.01 & 7.60 & 8. 50 & 7.12 & 7.62 & 10.78 & 7.21 & 7.98 & 9.83 & 8.41 & 9.43 & 8.84 & 9.05 \\
\hline 10 & 2.55 & 2.48 & 2.67 & 2.00 & 2.30 & 1.81 & 2.24 & 1.28 & 1.31 & 1.26 & 1.43 & 1.76 & 1.65 & 1.42 & 0.98 & 1.38 & 1.30 \\
\hline 11 & 56 & .36 & .86 & .80 & .50 & .56 & 1.18 & 1.14 & 1.24 & 88 & .88 & 1.07 & $N \cdot C$ & .95 & .65 & .95 & .83 \\
\hline 12 & 3.23 & 2.80 & 456 & 2.91 & 4.98 & 4.94 & 4.68 & 4.25 & 5.08 & 8.15 & 4.23 & 4.53 & 6.37 & 3.25 & 5.95 & 405 & 9.87 \\
\hline 13 & 16.2 .5 & 10.53 & 28.62 & 10.36 & 16.47 & 18.30 & 13.84 & 11.87 & 28.65 & 19.00 & 13.20 & 13.73 & 19.13 & 12.06 & 2450 & 22.66 & 9.05 \\
\hline 14 & 2.85 & 2.90 & 3.00 & 2.70 & 2.75 & 2.78 & 3.56 & 3.03 & 3.61 & 3.02 & 3.19 & 3.44 & 3.61 & 3.11 & 2.89 & 3.61 & 3.33 \\
\hline
\end{tabular}

N.C $=$ No comparable

\section{UTO}

\begin{tabular}{|c|c|c|c|c|c|c|c|c|c|c|c|c|c|c|c|c|c|}
\hline \multicolumn{18}{|c|}{ 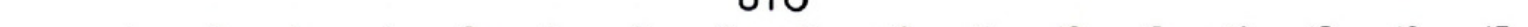 } \\
\hline & 1 & 2 & 3 & 4 & 5 & 6 & 7 & 8 & 9 & 10 & 11 & 12 & 13 & 14 & 15 & 16 & 17 \\
\hline 15 & 2.53 & 2.66 & 2.87 & 2.68 & 2.67 & 2.53 & 336 & 2.50 & 308 & 2.70 & 2.93 & 3.27 & 3.48 & 2.78 & 2.66 & 3.47 & 3.82 \\
\hline 16 & 1 & 1 & 1 & 1 & 1 & 1 & 2 & 2 & 2 & 2 & 2 & N.C & 2 & 2 & 2 & 2 & 2 \\
\hline 17 & 1.75 & 1.70 & 1.61 & 1.50 & 1.65 & 1.62 & 2.00 & 2.70 & 1.62 & 1.46 & UN.C & 1.88 & 1.97 & $\mathrm{N.C}$ & N.C & 1.71 & 1.88 \\
\hline 18 & 2.03 & 2.06 & 1.94 & 1.96 & 1.92 & 2.10 & 2. 37 & 2.50 & 2.01 & 1.99 & N.C & 2.12 & 2. 26 & N.C & N.C & 1.95 & 2.13 \\
\hline 19 & 2. 41 & 2.43 & 2.15 & 2.11 & 2.43 & 2.43 & 3.28 & 2.10 & 2.03 & 2.60 & N.C & 3.11 & 3.00 & 2. 91 & N.C & 2.88 & 2.96 \\
\hline 20 & 2.27 & 1.65 & 1.65 & 2.40 & 2.10 & 2.40 & 3. 27 & 300 & 2.66 & 2.43 & 2.62 & 368 & 3.20 & 2.61 & 3.46 & 3.53 & 3.12 \\
\hline 21 & 1.63 & 1. 20 & 1.20 & 1.69 & 1.54 & 1.72 & 2.77 & 2.05 & 2.04 & 1.72 & 2.03 & 2.97 & 2.40 & 1.93 & 2.25 & 2. 24 & 270 \\
\hline 22 & .87 & .92 & 1.01 & 1.00 & 1.25 & 1.35 & 2.07 & $2 . \infty$ & 1.80 & 1.62 & 1.55 & 2.30 & 1.99 & 1.34 & 1.91 & 1.83 & 2.25 \\
\hline 23 & 1 & 1 & 1 & 1 & 1 & 1 & 2 & N.C & 2 & 2 & N.C & 2 & 2 & $N \cdot C$ & N.C & $\varepsilon$ & 2 \\
\hline 24 & 4.97 & 3.60 & 2.63 & 6.16 & 8.90 & 8.67 & 11.21 & 8.50 & 8.61 & 14.12 & 7.27 & 11.74 & 11.99 & 7.27 & 7.45 & 5.92 & 944 \\
\hline 25 & 2.57 & 1.65 & 1.96 & 2.63 & 3.72 & 3.27 & 5.67 & 3.93 & 3.90 & 6.12 & 3.79 & 4.84 & 5.70 & 3.35 & 3.62 & 3.78 & 5.26 \\
\hline 26 & 3.40 & 2.90 & 249 & 2.47 & N.C & 2.90 & 3.77 & $\mathrm{~N} \cdot \mathrm{C}$ & 3.16 & 3.08 & 5.09 & 2. 81 & 4.30 & 3.02 & N.C & N.C & N.C \\
\hline 27 & 2.31 & 1.15 & 2.91 & 2.58 & 141 & 2.38 & 2.68 & 3.10 & 2.43 & 3.12 & 1.15 & 3.14 & 2.30 & 1.78 & 3.68 & 2.70 & 2.56 \\
\hline 28 & 1.70 & 1.30 & 1.18 & 1.84 & 1.20 & 1.66 & 2.04 & 1.55 & 1.12 & 1.16 & 1.08 & 1.45 & 1.87 & 1.99 & 2.27 & 2.62 & 2.15 \\
\hline
\end{tabular}




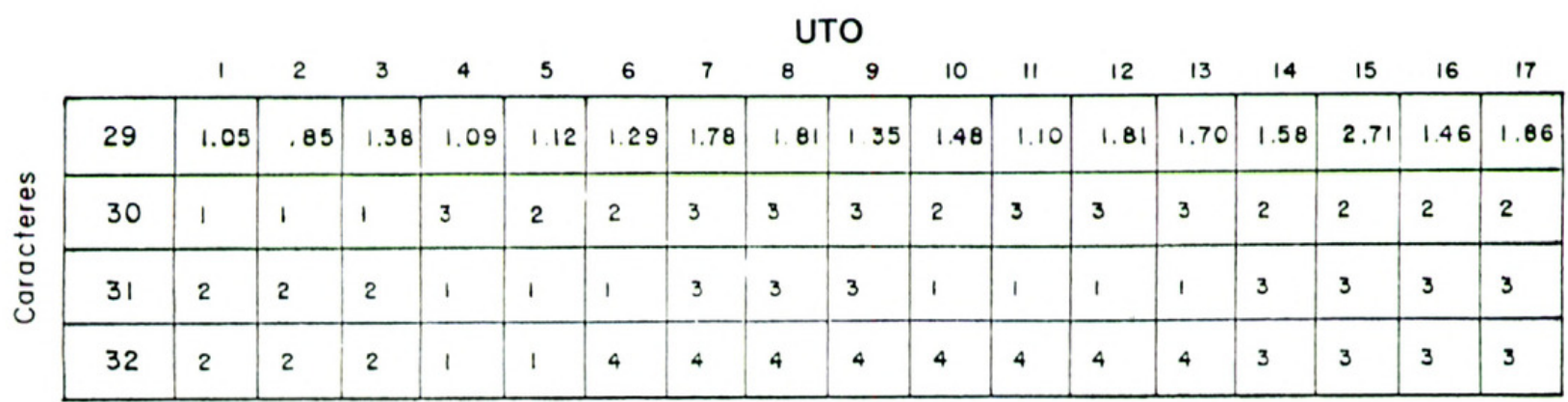




\section{$2 \mathrm{BHL}$ Biodiversity Heritage Library}

Orozco Pardo, Clara Inés. 1991. "Analisis Multivariado del Complejo de Tres Especies de Brunellia (Brunelliaceae)." Annals of the Missouri Botanical Garden 78, 970-994. https://doi.org/10.2307/2399738.

View This Item Online: https://www.biodiversitylibrary.org/item/89022

DOI: https://doi.org/10.2307/2399738

Permalink: https://www.biodiversitylibrary.org/partpdf/35625

\section{Holding Institution}

Missouri Botanical Garden, Peter H. Raven Library

\section{Sponsored by}

Missouri Botanical Garden

\section{Copyright \& Reuse}

Copyright Status: In copyright. Digitized with the permission of the rights holder.

License: http://creativecommons.org/licenses/by-nc-sa/3.0/

Rights: https://biodiversitylibrary.org/permissions

This document was created from content at the Biodiversity Heritage Library, the world's largest open access digital library for biodiversity literature and archives. Visit BHL at https://www.biodiversitylibrary.org. 